\title{
Basic variables to be reproduced in the first-principles theory for superconductors: Fluctuation of the particle number
}

\author{
Katsuhiko Higuchi \\ Graduate School of Advanced Sciences of Matter, \\ Hiroshima University, Higashi-Hiroshima 739-8527, Japan \\ Masahiko Higuchi \\ Department of Physics, Faculty of Science, \\ Shinshu University, Matsumoto 390-8621, Japan
}

(Dated: June 9, 2021)

\begin{abstract}
We show that the diagonal elements of the second-order reduced density matrix (RDM2) can be chosen as basic variables for describing the superconducting state, instead of the off-diagonal elements of the RDM2 that are usually adopted as basic variables in the density functional scheme. The diagonal elements of the RDM2 are called pair-density (PD), which is explicitly related to the fluctuation of the particle number of the system. In this paper, we argue that the fluctuation of the particle number can become an indication of the superconducting state, and that the density functional scheme in which the PD is chosen as a basic variable would be a promising first-principles theory for superconductors.
\end{abstract}

PACS numbers: $74.20 . \mathrm{Pq}$

Keywords: superconductivity, superconducting state, second-order reduced density matrix, fluctuation of the particle number, order parameter, pair-density, pair-density functional theory 


\section{INTRODUCTION}

The superconducting state of the fermion system is defined as the condensation of electrons into the same two-particle state, which is so-called the Bose-Einstein condensation (BEC) of the fermion system [1]. According to this definition, the off-diagonal elements of the second-order reduced density matrix (RDM2) of the system is related to the order parameter of the superconducting state (OPSS) [1 4]. The OPSS is sometimes called the offdiagonal long-range order [2-4]. The OPSS explicitly includes the two-particle wave function which forms the BEC, and accordingly reflects its spatial and spin symmetries rigorously [2, 3, 5]. Since these information are useful for understanding the properties of the superconductor, the first-principles theories have been developed for the purpose of calculating directly the OPSS [6 10]. Especially in the field of the density functional theory (DFT), the research has been active since the first theory presented by Oliveira, Gross and Kohn $\left[9\right.$, 10]. A lot of works including the actual applications to various superconductors $\left[\begin{array}{llll}11 & 29\end{array}\right]$ have been done so far. In all these works, the OPSS, i.e., off-diagonal elements of the RDM2, are certainly adopted as the basic variables that describe the equilibrium properties of the superconducting state $[11-29]$.

Thus the previous DFT-based theories for superconductors focuses on the off-diagonal elements of the RDM2 [11 29]. On the other hand, as shown in this paper, there exists the mutual relation between the off-diagonal elements of the RDM2 and the diagonal ones in the superconducting state. In other words, the off-diagonal elements of the RDM2 and the diagonal ones are not independent of each other in the superconducting state. This means that it is not necessary to use the off-diagonal elements of the RDM2 so as to describe the superconducting state, but that it is possible to use the diagonal elements instead of them. The diagonal elements of the RDM2 are called the pair-density (PD), which is explicitly related to the fluctuation of the particle number (Sec. IV). In this paper we will show that the fluctuation of the particle number, i.e., diagonal elements of the RDM2, can become an indication of whether the superconducting state appears or not in the system.

As a promising theory to predict the fluctuation of the particle number, we come up with the PD functional theory [30 60]. This is because the fluctuation of the particle number is calculated rigorously with the use of the PD and electron density, which will also be shown in the subsequent sections. In addition to the above reason, there would be an advantageous 
reason of developing the PD functional theory from another point of view. That is, it can be expected to utilize the findings and knowledge obtained in developing zero-temperature PD functional theory for the normal state material [30 60]. The zero-temperature PD functional theory has recently attracted much attention due to the reason that the PD contains full of the information on the static electron correlation [32]. Various approaches have attempted to overcome or avoid the problems of zero-temperature PD functional theory [30 60]. Especially, so-called the "scaling method" which is an effective method of searching PDs [37, 39], and the coupling-constant expression for the kinetic energy functional [38, 40] seem to be useful for the case of the superconductor. The finite-temperature PD functional theory for superconductors, which can predict the fluctuation of the particle number, has not yet proposed so far. In this paper, we present the theoretical framework of such the PD functional theory.

Organization of this paper is as follows. The superconducting state is defined as the BEC of the fermion system. Using this definition, the OPSS is reviewed in Sec. II for the convenience of the subsequent discussions. In Sec. III, we show that the fluctuation of the particle number becomes $O(N)$ when the OPSS appears in the system. This means that there exists another possible way to choose basic variables for describing the superconducting state. In order to develop the first-principles theory for reproducing the fluctuation of the particle number, in Sec. IV, we present the PD functional theory that is applicable to the superconductor. In Sec. V, some concluding remarks are presented together with the discussions on the signification of this PD functional theory.

\section{ORDER PARAMETER OF THE SUPERCONDUCTING STATE}

The superconducting state is defined with the aid of the concept of the BEC of the interacting fermion system [1]. In this section, we shall review the OPSS [2 4] with emphasize on the spatial broadenings and occupation numbers of two-particle states which form the BEC. These are essential for the definition of the OPSS. 


\section{A. Spectrum decomposition of the RDM2}

In order to define the superconducting state, it is necessary to consider two-particle states which are included in the RDM2 of the many-electron system. The operator of the RDM2 is given by [61]

$$
\hat{D}^{(2)}\left(\mathbf{r}_{1} \zeta_{1}, \mathbf{r}_{2} \zeta_{2} ; \mathbf{r}_{1}^{\prime} \zeta_{1}^{\prime}, \mathbf{r}_{2}^{\prime} \zeta_{2}^{\prime}\right)=\frac{1}{2} \psi^{\dagger}\left(\mathbf{r}_{1}^{\prime} \zeta_{1}^{\prime}\right) \psi^{\dagger}\left(\mathbf{r}_{2}^{\prime} \zeta_{2}^{\prime}\right) \psi\left(\mathbf{r}_{2} \zeta_{2}\right) \psi\left(\mathbf{r}_{1} \zeta_{1}\right)
$$

where $\psi\left(\mathbf{r}_{1} \zeta_{1}\right)$ and $\psi^{\dagger}\left(\mathbf{r}_{1} \zeta_{1}\right)$ are field operators of electrons, and $\mathbf{r}$ and $\zeta$ are spatial and spin coordinates, respectively. If the RDM2 of the system of state $|\Psi\rangle$ is denoted as $\left\langle\hat{D}^{(2)}\left(\mathbf{r}_{1} \zeta_{1}, \mathbf{r}_{2} \zeta_{2} ; \mathbf{r}_{1}^{\prime} \zeta_{1}^{\prime}, \mathbf{r}_{2}^{\prime} \zeta_{2}^{\prime}\right)\right\rangle_{\Psi}$, it is given by

$$
\begin{aligned}
\left\langle\hat{D}^{(2)}\left(\mathbf{r}_{1} \zeta_{1}, \mathbf{r}_{2} \zeta_{2} ; \mathbf{r}_{1}^{\prime} \zeta_{1}^{\prime}, \mathbf{r}_{2}^{\prime} \zeta_{2}^{\prime}\right)\right\rangle_{\Psi} & =\left\langle\Psi\left|\hat{D}^{(2)}\left(\mathbf{r}_{1} \zeta_{1}, \mathbf{r}_{2} \zeta_{2} ; \mathbf{r}_{1}^{\prime} \zeta_{1}^{\prime}, \mathbf{r}_{2}^{\prime} \zeta_{2}^{\prime}\right)\right| \Psi\right\rangle \\
& =\frac{1}{2}\left\langle\Psi\left|\psi^{\dagger}\left(\mathbf{r}_{1}^{\prime} \zeta_{1}^{\prime}\right) \psi^{\dagger}\left(\mathbf{r}_{2}^{\prime} \zeta_{2}^{\prime}\right) \psi\left(\mathbf{r}_{2} \zeta_{2}\right) \psi\left(\mathbf{r}_{1} \zeta_{1}\right)\right| \Psi\right\rangle
\end{aligned}
$$

Using Eq. (2), we shall define the operator $\hat{D}_{\Psi}^{(2)}$ as follows:

$$
\left\langle\mathbf{r}_{1} \zeta_{1}, \mathbf{r}_{2} \zeta_{2}\left|\hat{D}_{\Psi}^{(2)}\right| \mathbf{r}_{1}^{\prime} \zeta_{1}^{\prime}, \mathbf{r}_{2}^{\prime} \zeta_{2}^{\prime}\right\rangle=2\left\langle\hat{D}^{(2)}\left(\mathbf{r}_{1} \zeta_{1}, \mathbf{r}_{2} \zeta_{2} ; \mathbf{r}_{1}^{\prime} \zeta_{1}^{\prime}, \mathbf{r}_{2}^{\prime} \zeta_{2}^{\prime}\right)\right\rangle_{\Psi},
$$

where, as $|0\rangle$ is the vacuum state, the ket vector $\left|\mathbf{r}_{1} \zeta_{1}, \mathbf{r}_{2} \zeta_{2}\right\rangle$ is given by

$$
\left|\mathbf{r}_{1} \zeta_{1}, \mathbf{r}_{2} \zeta_{2}\right\rangle=\psi^{\dagger}\left(\mathbf{r}_{1} \zeta_{1}\right) \psi^{\dagger}\left(\mathbf{r}_{2} \zeta_{2}\right)|0\rangle
$$

If the eigenfunction and eigenvalue for the operator $\hat{D}_{\Psi}^{(2)}$ are denoted as $\left|\nu_{\Psi}\right\rangle$ and $n_{\nu_{\Psi}}^{(2)}$, respectively, the eigenvalue equation for $\hat{D}_{\Psi}^{(2)}$ is expressed as

$$
\hat{D}_{\Psi}^{(2)}\left|\nu_{\Psi}\right\rangle=n_{\nu_{\Psi}}^{(2)}\left|\nu_{\Psi}\right\rangle
$$

The eigenfunctions $\left|\nu_{\Psi}\right\rangle$ are two-particle states, which can be formally defined corresponding to the many-electron state $|\Psi\rangle$. They are sometimes called geminal in the field of the quantum chemistry [61]. We suppose that they form the orthonormal and complete set. Using these eigenfunctions and eigenvalues, the spectrum decomposition of the RDM2 is written as

$$
\left\langle\hat{D}^{(2)}\left(\mathbf{r}_{1} \zeta_{1}, \mathbf{r}_{2} \zeta_{2} ; \mathbf{r}^{\prime}{ }_{1} \zeta_{1}^{\prime}, \mathbf{r}^{\prime}{ }_{2} \zeta_{2}^{\prime}\right)\right\rangle_{\Psi}=\sum_{\nu_{\Psi}} n_{\nu_{\Psi}}^{(2)} \nu_{\Psi}\left(\mathbf{r}_{1} \zeta_{1}, \mathbf{r}_{2} \zeta_{2}\right) \nu_{\Psi}\left(\mathbf{r}_{1}^{\prime} \zeta_{1}^{\prime}, \mathbf{r}_{2}^{\prime} \zeta_{2}^{\prime}\right)^{*}
$$

where $\nu_{\Psi}\left(\mathbf{r}_{1} \zeta_{1}, \mathbf{r}_{2} \zeta_{2}\right)$ is the coordinate representation of two-particle state $\left|\nu_{\Psi}\right\rangle$, which is given by $\nu_{\Psi}\left(\mathbf{r}_{1} \zeta_{1}, \mathbf{r}_{2} \zeta_{2}\right)=\left\langle\mathbf{r}_{1} \zeta_{1}, \mathbf{r}_{2} \zeta_{2} \mid \nu_{\Psi}\right\rangle / \sqrt{2}$. The spatial broadening of $\nu_{\Psi}\left(\mathbf{r}_{1} \zeta_{1}, \mathbf{r}_{2} \zeta_{2}\right)$ is 
intimately associated with whether the system contains the pairing state such as the Cooper pair of the superconducting state or not. The magnitude of $n_{\nu_{\Psi}}^{(2)}$ is also significant for judging whether the superconducting state appears or not, because $n_{\nu_{\Psi}}^{(2)}$ corresponds to the occupation number of two-particle state $\nu_{\Psi}\left(\mathbf{r}_{1} \zeta_{1}, \mathbf{r}_{2} \zeta_{2}\right)$ [2]. The maximum value of $n_{\nu_{\Psi}}^{(2)}$ is also shown to be not $O\left(N^{2}\right)$ but $O(N)$ [1]. As will be discussed in the following subsection, both the spatial broadening of $\nu_{\Psi}\left(\mathbf{r}_{1} \zeta_{1}, \mathbf{r}_{2} \zeta_{2}\right)$ and the magnitude of $n_{\nu_{\Psi}}^{(2)}$ are key quantities for the definition of the superconducting state.

\section{B. Definition of the superconductivity}

Using the coordinate of center of gravity $\mathbf{R}$ and relative coordinate $\boldsymbol{\rho}$, where $\mathbf{R}=$ $\left(\mathbf{r}_{1}+\mathbf{r}_{2}\right) / 2, \boldsymbol{\rho}=\mathbf{r}_{1}-\mathbf{r}_{2}$, two-particle state (geminal) is rewritten as $\nu_{\Psi}\left(\mathbf{R} \boldsymbol{\rho} ; \zeta_{1} \zeta_{2}\right)$ instead of $\nu_{\Psi}\left(\mathbf{r}_{1} \zeta_{1}, \mathbf{r}_{2} \zeta_{2}\right)$. Let us consider two types of geminals in terms of their spatial broadenings. The first type is (a) geminal which is spatially extended with respect to both $\mathbf{R}$ and $\boldsymbol{\rho}$. In this case, the magnitude of the geminal can be estimated as

$$
\nu_{\Psi}\left(\mathbf{R} \boldsymbol{\rho} ; \zeta_{1} \zeta_{2}\right) \sim \frac{1}{\sqrt{\Omega}} \frac{1}{\sqrt{\Omega}}
$$

where $\Omega$ is the volume of the system. The second type is (b) geminal which is extended with respect to $\mathbf{R}$, but is localized with respect to $\boldsymbol{\rho}$ in some region $\omega(\ll \Omega)$. In this case, the magnitude of the geminal can be estimated as

$$
\nu_{\Psi}\left(\mathbf{R} \boldsymbol{\rho} ; \zeta_{1} \zeta_{2}\right) \sim \frac{1}{\sqrt{\Omega}} \frac{1}{\sqrt{\omega}}
$$

In case (a), there would not exist an attractive force between electrons, and any kinds of electron pairings do not form in the system. That is to say, the system is not under the superconducting state but under the normal state. On the other hand, in case (b), electrons would attract each other via some attractive force that overcomes the Coulomb repulsive force, which yields the pairings of electrons.

The BEC of the fermion system is defined by means of the spectrum decomposition of the RDM2 [1]. If the eigenvalue for some geminal becomes $O(N)$, namely, if the occupation number of some geminal becomes $O(N)$, then it is said that the BEC occurs in the fermion system [1, 2]. If such an eigenvalue and corresponding geminal are denoted as $n_{\nu_{\Psi}^{\max }}^{(2)}$ and $\left|\nu_{\Psi}^{\max }\right\rangle$, respectively, the spectrum decomposition ([6) is formally rewritten as 


$$
\begin{aligned}
\left\langle\hat{D}^{(2)}\left(\mathbf{r}_{1} \zeta_{1}, \mathbf{r}_{2} \zeta_{2} ; \mathbf{r}_{1}^{\prime} \zeta_{1}^{\prime}, \mathbf{r}_{2}^{\prime} \zeta_{2}^{\prime}\right)\right\rangle_{\Psi} & =n_{\nu_{\Psi}^{\max }}^{(2)} \nu_{\Psi}^{\max }\left(\mathbf{R} \boldsymbol{\rho} ; \zeta_{1} \zeta_{2}\right) \nu_{\Psi}^{\max }\left(\mathbf{R}^{\prime} \boldsymbol{\rho}^{\prime} ; \zeta_{1}^{\prime} \zeta_{2}^{\prime}\right)^{*} \\
& +\sum_{\nu_{\Psi} \neq \nu_{\Psi}^{\max }} n_{\nu_{\Psi}}^{(2)} \nu_{\Psi}\left(\mathbf{R} \boldsymbol{\rho} ; \zeta_{1} \zeta_{2}\right) \nu_{\Psi}\left(\mathbf{R}^{\prime} \boldsymbol{\rho}^{\prime} ; \zeta_{1}^{\prime} \zeta_{2}^{\prime}\right)^{*}
\end{aligned}
$$

Let us assume that the BEC originates from only one kind of geminal, i.e., $\left|\nu_{\Psi}^{\max }\right\rangle[62]$. Then, the eigenvalues of any geminals except $\left|\nu_{\Psi}^{\max }\right\rangle$ are much less than $O(N)$, and the spatial broadenings of these geminals, i.e., $\nu_{\Psi}\left(\mathbf{r}_{1} \zeta_{1}, \mathbf{r}_{2} \zeta_{2}\right)$ 's that appear in the second term of Eq. (9), are necessarily type (a). This is because if some geminal other than $\left|\nu_{\Psi}^{\max }\right\rangle$ belongs to type (b) the BEC associated with it would appear simultaneously with the BEC of $\left|\nu_{\Psi}^{\max }\right\rangle$ [63], which contradicts the above assumption.

Here we shall give the definition of the superconducting state. When the BEC occurs with some geminal, and further when such a geminal is type (b), then we will say that the superconducting state appears in the system. This definition sounds reasonable, because the number of pairings is $O(N)$ and they are occupied in the same two-particle state, which is analogous with the intuitive description of the BEC of the ideal bosons, and further because such two-particle state is spatially localized like the Cooper pair [64, 65]. Thus, in the superconducting state, we have

$$
\left\{\begin{aligned}
n_{\nu_{\Psi}^{\max }}^{(2)}=O(N), & \nu_{\Psi}^{\max }\left(\mathbf{r}_{1} \zeta_{1}, \mathbf{r}_{2} \zeta_{2}\right)=\operatorname{type}(\mathrm{b}), \\
n_{\nu_{\Psi}}^{(2)} & \ll O(N), \quad \nu_{\Psi}\left(\mathbf{r}_{1} \zeta_{1}, \mathbf{r}_{2} \zeta_{2}\right)=\operatorname{type}(\mathrm{a}),
\end{aligned}\right.
$$

The striking point of the present definition is to define the spatial broadening of geminals which are condensed in the superconducting state.

\section{Order parameter of the superconducting state}

In this subsection, according to the definition of the superconducting state, we shall revisit the OPSS. As mentioned in Sec. II-B, the eigenvalue of some geminal becomes $O(N)$ when the superconducting state appears in the system.

First, let us take the following limit on both sides of Eq. (99) [1]:

$$
\mathbf{r}_{1} \approx \mathbf{r}_{2}, \quad \mathbf{r}_{1}^{\prime} \approx \mathbf{r}_{2}^{\prime} \text {, and }\left|\mathbf{r}_{1}-\mathbf{r}_{1}^{\prime}\right| \longrightarrow \infty
$$

This limit means that the distance between particles belonging to the same geminal gets close to each other and the distance between geminals spreads infinitely. It should be noted 
that the limit (11) includes the thermodynamical limit given by

$$
N \rightarrow \infty, \Omega \rightarrow \infty, \text { with } \frac{N}{\Omega}=n \text { (constant) }
$$

where $N$ and $\Omega$ are particle number and volume of the system, respectively. In the superconducting state, the first term of the right-hand side of Eq. (9) remains and takes the following value when taking the limit of Eq. (11):

$$
n_{\nu_{\Psi}^{\max }}^{(2)} \nu_{\Psi}^{\max }\left(\mathbf{R} \boldsymbol{\rho} ; \zeta_{1} \zeta_{2}\right) \nu_{\Psi}^{\max }\left(\mathbf{R}^{\prime} \boldsymbol{\rho}^{\prime} ; \zeta_{1}^{\prime} \zeta_{2}^{\prime}\right)^{*} \approx O(N)\left(\frac{1}{\sqrt{\Omega}} \frac{1}{\sqrt{\omega}}\right)^{2}
$$

where Eqs. (10) and (8) are used. Equation (13) takes the value of the order of $n / \omega$, which is a finite and nonzero value. On the other hand, the second term of Eq. (9) is shown to be zero in the limit of Eq. (11) when the system is under the superconducting state. It is proved as follows.

Since we merely intend to check whether the second term of Eq. (9) vanish or not in the limit of Eq. (11), it is sufficient to estimate the order of the magnitude of $\nu_{\Psi}\left(\mathbf{R} \boldsymbol{\rho} ; \zeta_{1} \zeta_{2}\right)$. For this aim, it seems to be appropriate to adopt the Slater determinant as an explicit form of $\nu_{\Psi}\left(\mathbf{R} \boldsymbol{\rho} ; \zeta_{1} \zeta_{2}\right)$. In other words, the Hartree-Fock approximation may be used for the order estimation of the magnitude of $\nu_{\Psi}\left(\mathbf{R} \boldsymbol{\rho} ; \zeta_{1} \zeta_{2}\right)$. A similar estimation using the Hartree-Fock approximation has been done by Yang in the paper where the BEC of the fermion system was first discussed [1]. Since $\nu_{\Psi}\left(\mathbf{R} \boldsymbol{\rho} ; \zeta_{1} \zeta_{2}\right)$ is spatially extended with respect to both $\mathbf{R}$ and $\boldsymbol{\rho}$, as mentioned in Eq. (10), it is appropriate to suppose that the Slater determinant is constructed from the plane waves. Namely we have

$$
\begin{aligned}
\nu_{\Psi}\left(\mathbf{R} \boldsymbol{\rho} ; \zeta_{1} \zeta_{2}\right) & =\nu_{\Psi}\left(\mathbf{r}_{1} \zeta_{1}, \mathbf{r}_{2} \zeta_{2}\right) \\
& =\frac{1}{\sqrt{2}}\left|\begin{array}{ll}
\phi_{a}\left(\mathbf{r}_{1} \zeta_{1}\right) & \phi_{b}\left(\mathbf{r}_{1} \zeta_{1}\right) \\
\phi_{a}\left(\mathbf{r}_{2} \zeta_{2}\right) & \phi_{b}\left(\mathbf{r}_{2} \zeta_{2}\right)
\end{array}\right|
\end{aligned}
$$

where $\phi_{a}(\mathbf{r} \zeta)$ is the plane wave containing the spin function $\chi_{\sigma_{a}}(\zeta)$, which is given by

$$
\phi_{a}(\mathbf{r} \zeta)=\frac{1}{\sqrt{\Omega}} e^{i \mathbf{k}_{a} \cdot \mathbf{r}} \chi_{\sigma_{a}}(\zeta)
$$

Substituting Eq. (15) into Eq. (14), we get

$$
\nu_{\Psi}\left(\mathbf{R} \boldsymbol{\rho} ; \zeta_{1} \zeta_{2}\right)=\frac{1}{\sqrt{2} \Omega} e^{i\left(\mathbf{k}_{a}+\mathbf{k}_{b}\right) \cdot \mathbf{R}}\left\{e^{i\left(\mathbf{k}_{a}-\mathbf{k}_{b}\right) \cdot \frac{\rho}{2}} \chi_{\sigma_{a}}\left(\zeta_{1}\right) \chi_{\sigma_{b}}\left(\zeta_{2}\right)-e^{-i\left(\mathbf{k}_{a}-\mathbf{k}_{b}\right) \cdot \frac{\rho}{2}} \chi_{\sigma_{a}}\left(\zeta_{2}\right) \chi_{\sigma_{b}}\left(\zeta_{1}\right)\right\}
$$


Note that Eq. (16) is the spatially-extended function with respect to both $\mathbf{R}$ and $\boldsymbol{\rho}$.

Using Eq. (16), the second term of Eq. (9) is expressed as

$$
\begin{aligned}
& \sum_{\nu_{\Psi} \neq \nu_{\Psi}^{\max }} n_{\nu_{\Psi}}^{(2)} \nu_{\Psi}\left(\mathbf{R} \boldsymbol{\rho} ; \zeta_{1} \zeta_{2}\right) \nu_{\Psi}\left(\mathbf{R}^{\prime} \boldsymbol{\rho}^{\prime} ; \zeta_{1}^{\prime} \zeta_{2}^{\prime}\right)^{*} \\
&=\sum_{\mathbf{k}_{a}, \mathbf{k}_{b}, \sigma_{a}, \sigma_{b}} \frac{n_{\mathbf{k}_{a} \sigma_{a} \mathbf{k}_{b} \sigma_{b}}^{(2)}}{2 \Omega}\left\{e^{i \mathbf{k}_{a} \cdot\left(\mathbf{r}_{1}-\mathbf{r}^{\prime} 1\right)} e^{i \mathbf{k}_{b} \cdot\left(\mathbf{r}_{2}-\mathbf{r}^{\prime}{ }_{2}\right)} \chi_{\sigma_{a}}\left(\zeta_{1}\right) \chi_{\sigma_{b}}\left(\zeta_{2}\right) \chi_{\sigma_{a}}\left(\zeta_{1}^{\prime}\right) \chi_{\sigma_{b}}\left(\zeta_{2}^{\prime}\right)\right. \\
&-e^{i \mathbf{k}_{a} \cdot\left(\mathbf{r}_{1}-\mathbf{r}_{2}^{\prime}\right)} e^{i \mathbf{k}_{b} \cdot\left(\mathbf{r}_{2}-\mathbf{r}^{\prime} 1\right)} \chi_{\sigma_{a}}\left(\zeta_{1}\right) \chi_{\sigma_{b}}\left(\zeta_{2}\right) \chi_{\sigma_{a}}\left(\zeta_{2}^{\prime}\right) \chi_{\sigma_{b}}\left(\zeta_{1}^{\prime}\right) \\
&-e^{i \mathbf{k}_{a} \cdot\left(\mathbf{r}_{2}-\mathbf{r}^{\prime} 1\right)} e^{i \mathbf{k}_{b} \cdot\left(\mathbf{r}_{1}-\mathbf{r}^{\prime} 2\right)} \chi_{\sigma_{a}}\left(\zeta_{2}\right) \chi_{\sigma_{b}}\left(\zeta_{1}\right) \chi_{\sigma_{a}}\left(\zeta_{1}^{\prime}\right) \chi_{\sigma_{b}}\left(\zeta_{2}^{\prime}\right) \\
&\left.+e^{i \mathbf{k}_{a} \cdot\left(\mathbf{r}_{2}-\mathbf{r}_{2}{ }_{2}\right)} e^{i \mathbf{k}_{b} \cdot\left(\mathbf{r}_{1}-\mathbf{r}^{\prime} 1\right)} \chi_{\sigma_{a}}\left(\zeta_{2}\right) \chi_{\sigma_{b}}\left(\zeta_{1}\right) \chi_{\sigma_{a}}\left(\zeta_{2}^{\prime}\right) \chi_{\sigma_{b}}\left(\zeta_{1}^{\prime}\right)\right\} .
\end{aligned}
$$

We shall take the limit of Eq. (11) on the right-hand side of Eq. (17). Using the RiemannLebesgue theorem for the oscillating function [66], all four terms are shown to vanish in this limit. Namely,

$$
\sum_{\nu_{\Psi} \neq \nu_{\Psi}^{\max }} n_{\nu_{\Psi}}^{(2)} \nu_{\Psi}\left(\mathbf{R} \boldsymbol{\rho} ; \zeta_{1} \zeta_{2}\right) \nu_{\Psi}\left(\mathbf{R}^{\prime} \boldsymbol{\rho}^{\prime} ; \zeta_{1}^{\prime} \zeta_{2}^{\prime}\right)^{*} \underset{\mathrm{Eq} \cdot(11)}{\longrightarrow} 0 .
$$

Considering Eqs. (13) and (18) together, the RDM2 $\left\langle\hat{D}^{(2)}\left(\mathbf{r}_{1} \zeta_{1}, \mathbf{r}_{2} \zeta_{2} ; \mathbf{r}_{1}^{\prime} \zeta_{1}^{\prime}, \mathbf{r}_{2}{ }_{2} \zeta_{2}^{\prime}\right)\right\rangle_{\Psi}$ can take a finite and nonzero value in the limit of Eq. (11) when the system $|\Psi\rangle$ is in the superconducting state. This statement is written by the following formula:

$$
\left\langle\hat{D}^{(2)}\left(\mathbf{r}_{1} \zeta_{1}, \mathbf{r}_{2} \zeta_{2} ; \mathbf{r}^{\prime}{ }_{1} \zeta_{1}^{\prime}, \mathbf{r}_{2}^{\prime} \zeta_{2}^{\prime}\right)\right\rangle_{\Psi} \underset{\mathbf{E q} \cdot(11)}{\longrightarrow} n_{\nu_{\Psi}^{\max }}^{(2)} \nu_{\Psi}^{\max }\left(\mathbf{R} \boldsymbol{\rho} ; \zeta_{1} \zeta_{2}\right) \nu_{\Psi}^{\max }\left(\mathbf{R}^{\prime} \boldsymbol{\rho}^{\prime} ; \zeta_{1}^{\prime} \zeta_{2}^{\prime}\right)^{*}
$$

Of course, the right-hand side of Eq. (19) is nonzero, and is estimated to be the order of $n / \omega$ in the superconducting state, as mentioned above. Equation (19) means that off-diagonal elements of the RDM2 of the superconducting state becomes the order of $n / \omega$ in the limit of Eq. (11).

On the other hand, let us consider the case of taking the limit of Eq. (11) directly on both sides of Eq. (2). Generally, there does not exist any correlation between two systems which separate from each other in an infinite distance [67]. If $\hat{A}(\mathbf{r})$ and $\hat{B}\left(\mathbf{r}^{\prime}\right)$ are arbitrary operators depending on the positions $\mathbf{r}$ and $\mathbf{r}^{\prime}$, respectively, and further if the distance between $\mathbf{r}$ and $\mathbf{r}^{\prime}$ is infinite, then the expectation value of the product of them is equal to the product of the individual expectation values:

$$
\left\langle\Psi\left|\hat{A}(\mathbf{r}) \hat{B}\left(\mathbf{r}^{\prime}\right)\right| \Psi\right\rangle \underset{\left|\mathbf{r}-\mathbf{r}^{\prime}\right| \rightarrow \infty}{\longrightarrow}\langle\Psi|\hat{A}(\mathbf{r})| \Psi\rangle\left\langle\Psi\left|\hat{B}\left(\mathbf{r}^{\prime}\right)\right| \Psi\right\rangle .
$$


Equation (20) is sometimes called the cluster decomposition principle [67]. When taking the limit of Eq. (11), we can investigate the behavior of the RDM2 in the case where the correlation between geminals does not exist at all. Using the general principle (20), Eq. (2) becomes

$$
\left\langle\hat{D}^{(2)}\left(\mathbf{r}_{1} \zeta_{1}, \mathbf{r}_{2} \zeta_{2} ; \mathbf{r}_{1}^{\prime} \zeta_{1}^{\prime}, \mathbf{r}_{2}^{\prime} \zeta_{2}^{\prime}\right)\right\rangle_{\Psi} \underset{\mathrm{Eq} \cdot(11)}{\longrightarrow} \frac{1}{2}\left\langle\Psi\left|\psi^{\dagger}\left(\mathbf{r}_{1}^{\prime} \zeta_{1}^{\prime}\right) \psi^{\dagger}\left(\mathbf{r}_{2}^{\prime} \zeta_{2}^{\prime}\right)\right| \Psi\right\rangle\left\langle\Psi\left|\psi\left(\mathbf{r}_{2} \zeta_{2}\right) \psi\left(\mathbf{r}_{1} \zeta_{1}\right)\right| \Psi\right\rangle .
$$

Compared Eq. (21) with Eq. (19), we immediately understand that the OPSS is given by $\left\langle\Psi\left|\psi(\mathbf{r} \zeta) \psi\left(\mathbf{r}^{\prime} \zeta^{\prime}\right)\right| \Psi\right\rangle$. Namely we get

$$
\left\langle\Psi\left|\psi(\mathbf{r} \zeta) \psi\left(\mathbf{r}^{\prime} \zeta^{\prime}\right)\right| \Psi\right\rangle=\sqrt{2 n_{\nu_{\Psi}^{\max }}^{(2)}} \nu_{\Psi}^{\max }\left(\mathbf{r} \zeta, \mathbf{r}^{\prime} \zeta^{\prime}\right)
$$

If $\left\langle\Psi\left|\psi(\mathbf{r} \zeta) \psi\left(\mathbf{r}^{\prime} \zeta^{\prime}\right)\right| \Psi\right\rangle$ takes a nonzero value, then the system $|\Psi\rangle$ is in the superconducting state.

Further from Eq. (22), the spatial and spin symmetries for the pairing states can be confirmed by means of the OPSS. That is to say, the OPSS $\left\langle\Psi\left|\psi(\mathbf{r} \zeta) \psi\left(\mathbf{r}^{\prime} \zeta^{\prime}\right)\right| \Psi\right\rangle$ is formally rewritten as a function of $\mathbf{R}$ and $\boldsymbol{\rho}$, which directly gives the spatial distribution of the pairing states via Eq. (22). Also concerning the spin symmetry, it is easily shown that the OPSS $\left\langle\Psi\left|\psi(\mathbf{r} \zeta) \psi\left(\mathbf{r}^{\prime} \zeta^{\prime}\right)\right| \Psi\right\rangle$ is decomposed into the parts of spin-singlet and spin-triplet wave functions.

\section{The case of the finite temperature}

Let us extend the above-mentioned discussions to the case of the finite temperature. The superconducting state should be treated on the basis of the grand canonical ensemble because the number of electrons that form the superconducting phase varies depending on the condition of the system. If the statistical density matrix of the grand canonical ensemble is denoted as $\hat{\rho}_{H}$, the statistical average of the physical quantity $\hat{A}$ is given by $\operatorname{Tr}\left(\hat{\rho}_{H} \hat{A}\right)$, which is hereafter denoted as $\langle\hat{A}\rangle_{H}$. The statistical average of the RDM2 with respect to $\hat{\rho}_{H}$ is given by

$$
\left\langle\hat{D}^{(2)}\left(\mathbf{r}_{1} \zeta_{1}, \mathbf{r}_{2} \zeta_{2} ; \mathbf{r}_{1}^{\prime} \zeta_{1}^{\prime}, \mathbf{r}_{2}^{\prime} \zeta_{2}^{\prime}\right)\right\rangle_{H}=\operatorname{Tr}\left\{\hat{\rho}_{H} \hat{D}^{(2)}\left(\mathbf{r}_{1} \zeta_{1}, \mathbf{r}_{2} \zeta_{2} ; \mathbf{r}_{1}^{\prime} \zeta_{1}^{\prime}, \mathbf{r}_{2}^{\prime} \zeta_{2}^{\prime}\right)\right\}
$$

In a similar way to the case of zero-temperature (Eq. (3) ), we can define the operator $\hat{D}_{H}^{(2)}$ with the use of the equation $\left\langle\mathbf{r}_{1} \zeta_{1} \mathbf{r}_{2} \zeta_{2}\left|\hat{D}_{H}^{(2)}\right| \mathbf{r}_{1}^{\prime} \zeta_{1}^{\prime} \mathbf{r}_{2}^{\prime} \zeta_{2}^{\prime}\right\rangle=2\left\langle\hat{D}^{(2)}\left(\mathbf{r}_{1} \zeta_{1}, \mathbf{r}_{2} \zeta_{2} ; \mathbf{r}^{\prime}{ }_{1}^{\prime} \zeta_{1}, \mathbf{r}_{2}^{\prime} \zeta_{2}^{\prime}\right)\right\rangle_{H}$. 
The eigenvalue equation for $\hat{D}_{H}^{(2)}$ is formally written as

$$
\hat{D}_{H}^{(2)}\left|\nu_{H}\right\rangle=n_{\nu_{H}}^{(2)}\left|\nu_{H}\right\rangle
$$

where $\left|\nu_{H}\right\rangle$ and $n_{\nu_{H}}^{(2)}$ are eigenfunctions and eigenvalues for $\hat{D}_{H}^{(2)}$, respectively. Using Eq. (24), the spectrum decomposition for $\left\langle\hat{D}^{(2)}\left(\mathbf{r}_{1} \zeta_{1}, \mathbf{r}_{2} \zeta_{2} ; \mathbf{r}_{1}^{\prime} \zeta_{1}^{\prime}, \mathbf{r}_{2}^{\prime} \zeta_{2}^{\prime}\right)\right\rangle_{H}$ is given by

$$
\left\langle\hat{D}^{(2)}\left(\mathbf{r}_{1} \zeta_{1}, \mathbf{r}_{2} \zeta_{2} ; \mathbf{r}^{\prime}{ }_{1} \zeta_{1}^{\prime}, \mathbf{r}_{2}^{\prime} \zeta_{2}^{\prime}\right)\right\rangle_{H}=\sum_{\nu_{H}} n_{\nu_{H}}^{(2)} \nu_{H}\left(\mathbf{r}_{1} \zeta_{1}, \mathbf{r}_{2} \zeta_{2}\right) \nu_{H}\left(\mathbf{r}_{1}^{\prime} \zeta_{1}^{\prime}, \mathbf{r}_{2}^{\prime} \zeta_{2}^{\prime}\right)^{*}
$$

in a similar way to Eq. (6)).

The superconducting state can be defined in the same way as the case of zero-temperature (Sec. II-B). Specifically, the definition of the superconducting state is given by the following replacement in Eq. (10):

$$
\left\{\begin{array} { c } 
{ n _ { \nu _ { \Psi } } ^ { ( 2 ) } } \\
{ n _ { \nu _ { \Psi } ^ { \operatorname { m a x } } } ^ { ( 2 ) } } \\
{ \nu _ { \Psi } ( \mathbf { r } _ { 1 } \zeta _ { 1 } , \mathbf { r } _ { 2 } \zeta _ { 2 } ) } \\
{ \nu _ { \Psi } ^ { \operatorname { m a x } } ( \mathbf { r } _ { 1 } \zeta _ { 1 } , \mathbf { r } _ { 2 } \zeta _ { 2 } ) }
\end{array} \longrightarrow \left\{\begin{array}{c}
n_{\nu_{H}}^{(2)} \\
n_{\nu_{H}^{\max }}^{(2)} \\
\nu_{H}\left(\mathbf{r}_{1} \zeta_{1}, \mathbf{r}_{2} \zeta_{2}\right) \\
\nu_{H}^{\max }\left(\mathbf{r}_{1} \zeta_{1}, \mathbf{r}_{2} \zeta_{2}\right)
\end{array}\right.\right.
$$

Also concerning the OPSS, we can obtain it by using Eq. (26) and by replacing the expectation value $\langle\Psi|\hat{A}| \Psi\rangle$ with the statistical average $\langle\hat{A}\rangle_{H}$ in the discussions of Sec. II-C. The explicit form of the OPSS at the finite temperature is given by

$$
\left\langle\psi(\mathbf{r} \zeta) \psi\left(\mathbf{r}^{\prime} \zeta^{\prime}\right)\right\rangle_{H}=\operatorname{Tr}\left\{\hat{\rho}_{H} \psi(\mathbf{r} \zeta) \psi\left(\mathbf{r}^{\prime} \zeta^{\prime}\right)\right\}
$$

Here we suppose that the cluster decomposition principle holds also at the finite temperature if the system is stable [67]:

$$
\left\langle\hat{A}(\mathbf{r}) \hat{B}\left(\mathbf{r}^{\prime}\right)\right\rangle_{H} \underset{\left|\mathbf{r}-\mathbf{r}^{\prime}\right| \rightarrow \infty}{\longrightarrow}\langle\hat{A}(\mathbf{r})\rangle_{H}\left\langle\hat{B}\left(\mathbf{r}^{\prime}\right)\right\rangle_{H},
$$

where $\hat{A}(\mathbf{r})$ and $\hat{B}\left(\mathbf{r}^{\prime}\right)$ are operators of arbitrary physical quantities. In a similar way to Eq. (22), the OPSS (27) is rewritten as

$$
\left\langle\psi(\mathbf{r} \zeta) \psi\left(\mathbf{r}^{\prime} \zeta^{\prime}\right)\right\rangle_{H}=\sqrt{2 n_{\nu_{H}^{\max }}^{(2)}} \nu_{H}^{\max }\left(\mathbf{r} \zeta, \mathbf{r}^{\prime} \zeta^{\prime}\right) .
$$

Thus, the extension to the finite temperature can be done by the replacement

$$
\left\langle\Psi\left|\psi(\mathbf{r} \zeta) \psi\left(\mathbf{r}^{\prime} \zeta^{\prime}\right)\right| \Psi\right\rangle \quad \longrightarrow \quad\left\langle\psi(\mathbf{r} \zeta) \psi\left(\mathbf{r}^{\prime} \zeta^{\prime}\right)\right\rangle_{H}
$$

in addition to Eq. (26). 


\section{FLUCTUATIONS OF THE PARTICLE NUMBER IN THE SUPERCON- DUCTING STATE}

In this section, it is shown that diagonal elements of the RDM2 can be used as an indication quantity of the superconducting state instead of the conventionally-used OPSS, i.e., off-diagonal elements of the RDM2. Specifically, we shall show that the fluctuation of the particle number becomes $O(N)$ when the OPSS appears in the system.

\section{A. Bloch-de Dominicis theorem for the RDM2}

It is sufficient for the order estimation of the physical quantities to make a use of the Hartree-Fock approximation. In Sec.II-C, we have already adopted the Hartree-Fock approximation for estimating the order of the second term of Eq. (9). In this subsection we shall consider the Hartree-Fock approximation of the RDM2 at finite temperature in the superconducting state.

The Hartree-Fock approximation of some physical quantity at the finite temperature corresponds to the statistical average of the quantity by means of the density matrix involving the mean-field Hamiltonian. In the superconducting state, the mean-field Hamiltonian is devised such that the gauge symmetry is explicitly broken, as can be seen in the BCS theory [65], Bogoliubov-de Gennes theory [7, 8], and the density-functional schemes [9, 10, 25]. If such a mean-field Hamiltonian is denoted as $\hat{H}_{M}$, then it does not preserve the electron number, but can be diagonalized in terms of the quasiparticles via the Bogoliubov-Valatin transformation [68, 69]. The Bogoliubov-Valatin transformation from the electron system to the quasiparticle system is generally written as [68, 69]

$$
\begin{aligned}
& \psi(\mathbf{r} \zeta)=\sum_{i} u_{i}(\mathbf{r} \zeta) \gamma_{i}+\sum_{j} v_{j}(\mathbf{r} \zeta) \gamma_{j}^{\dagger} \\
& \psi^{\dagger}(\mathbf{r} \zeta)=\sum_{i} u_{i}^{*}(\mathbf{r} \zeta) \gamma_{i}^{\dagger}+\sum_{j} v_{j}^{*}(\mathbf{r} \zeta) \gamma_{j}
\end{aligned}
$$

where $\gamma_{i}$ and $\gamma_{i}^{\dagger}$ are annihilation and creation operators of quasiparticles, and where $u_{i}(\mathbf{r} \zeta)$ and $v_{i}(\mathbf{r} \zeta)$ are elements of the unitary matrix which are determined by requiring that $\hat{H}_{M}$ is diagonalized in terms of the quasiparticles [8]. Suppose that $\hat{H}_{M}$ is written as

$$
\hat{H}_{M}-\mu \hat{N}=\sum_{i} \xi_{i} \gamma_{i}^{\dagger} \gamma_{i}
$$


where $\hat{N}$ is the operator of the particle number, and where $\mu$ is the chemical potential of the system. By using this $\hat{H}_{M}$, we consider the statistical average of the RDM2, i.e., the Hartree-Fock approximation of the RDM2:

$$
\left\langle\hat{D}^{(2)}\left(\mathbf{r}_{4} \zeta_{4}, \mathbf{r}_{3} \zeta_{3} ; \mathbf{r}_{1} \zeta_{1}, \mathbf{r}_{2} \zeta_{2}\right)\right\rangle_{H_{M}}=\frac{1}{2} \operatorname{Tr}\left\{\hat{\rho}_{H_{M}} \psi^{\dagger}\left(\mathbf{r}_{1} \zeta_{1}\right) \psi^{\dagger}\left(\mathbf{r}_{2} \zeta_{2}\right) \psi\left(\mathbf{r}_{3} \zeta_{3}\right) \psi\left(\mathbf{r}_{4} \zeta_{4}\right)\right\},
$$

where the concrete forms of the statistical density matrix $\hat{\rho}_{H_{M}}$ is given by

$$
\hat{\rho}_{H_{M}}=\frac{1}{\Xi} e^{-\beta\left(\hat{H}_{M}-\mu \hat{N}\right)}
$$

with $\Xi=\operatorname{Tr}\left(e^{-\beta\left(\hat{H}_{M}-\mu \hat{N}\right)}\right)$. Using Eqs. (31) , (32) and (34), it is shown that the following cluster decomposition of the RDM2, which is so-called the Bloch-de Dominicis theorem [70 72], holds:

$$
\begin{aligned}
\left\langle\psi^{\dagger}\left(\mathbf{r}_{1} \zeta_{1}\right) \psi^{\dagger}\left(\mathbf{r}_{2} \zeta_{2}\right) \psi\left(\mathbf{r}_{3} \zeta_{3}\right) \psi\left(\mathbf{r}_{4} \zeta_{4}\right)\right\rangle_{H_{M}} & =\left\langle\psi^{\dagger}\left(\mathbf{r}_{1} \zeta_{1}\right) \psi\left(\mathbf{r}_{4} \zeta_{4}\right)\right\rangle_{H_{M}}\left\langle\psi^{\dagger}\left(\mathbf{r}_{2} \zeta_{2}\right) \psi\left(\mathbf{r}_{3} \zeta_{3}\right)\right\rangle_{H_{M}} \\
& -\left\langle\psi^{\dagger}\left(\mathbf{r}_{1} \zeta_{1}\right) \psi\left(\mathbf{r}_{3} \zeta_{3}\right)\right\rangle_{H_{M}}\left\langle\psi^{\dagger}\left(\mathbf{r}_{2} \zeta_{2}\right) \psi\left(\mathbf{r}_{4} \zeta_{4}\right)\right\rangle_{H_{M}} \\
& +\left\langle\psi^{\dagger}\left(\mathbf{r}_{1} \zeta_{1}\right) \psi^{\dagger}\left(\mathbf{r}_{2} \zeta_{2}\right)\right\rangle_{H_{M}}\left\langle\psi\left(\mathbf{r}_{3} \zeta_{3}\right) \psi\left(\mathbf{r}_{4} \zeta_{4}\right)\right\rangle_{H_{M}}
\end{aligned}
$$

This equation means that the Bloch-de Dominicis theorem holds even when taking the statistical average of the RDM2 written by the field operators of electrons by means of the statistical density matrix of the noninteracting quasiparticle system.

\section{B. Idempotent of the RDM1}

On the right-hand side of Eq. (35), we have the statistical average of the first-order reduced density matrix (RDM1). For the convenience of the later discussion, let us consider the idempotent of the RDM1. As is well known, the idempotent of the RDM1 rigorously holds at zero temperature within the Hartree-Fock approximation [61]. However, it is not obvious that such the idempotent holds in the superconducting state at the finite temperature. To tell the conclusion first, the idempotent of the RDM1 approximately holds even in the superconducting state at low temperature. We will show it below.

The operator of the RDM1 is given by [61]

$$
\hat{D}^{(1)}\left(\mathbf{r}_{1} \zeta_{1}, \mathbf{r}_{2} \zeta_{2}\right)=\psi^{\dagger}\left(\mathbf{r}_{2} \zeta_{2}\right) \psi\left(\mathbf{r}_{1} \zeta_{1}\right)
$$


If the RDM1 of the system of state $|\Psi\rangle$ is denoted as $\left\langle\hat{D}^{(1)}\left(\mathbf{r}_{1} \zeta_{1}, \mathbf{r}_{2} \zeta_{2}\right)\right\rangle_{\Psi}$, it is given by

$$
\left\langle\hat{D}^{(1)}\left(\mathbf{r}_{1} \zeta_{1}, \mathbf{r}_{2} \zeta_{2}\right)\right\rangle_{\Psi}=\left\langle\Psi\left|\hat{D}^{(1)}\left(\mathbf{r}_{1} \zeta_{1}, \mathbf{r}_{2} \zeta_{2}\right)\right| \Psi\right\rangle
$$

Also if the statistical average of $\hat{D}^{(1)}\left(\mathbf{r}_{1} \zeta_{1}, \mathbf{r}_{2} \zeta_{2}\right)$ with the density matrix $\hat{\rho}_{H_{M}}$ is denoted as $\left\langle\hat{D}^{(1)}\left(\mathbf{r}_{1} \zeta_{1}, \mathbf{r}_{2} \zeta_{2}\right)\right\rangle_{H_{M}}$, then it is written as

$$
\left\langle\hat{D}^{(1)}\left(\mathbf{r}_{1} \zeta_{1}, \mathbf{r}_{2} \zeta_{2}\right)\right\rangle_{H_{M}}=\operatorname{Tr}\left(\hat{\rho}_{H_{M}} \hat{D}^{(1)}\left(\mathbf{r}_{1} \zeta_{1}, \mathbf{r}_{2} \zeta_{2}\right)\right)
$$

When the trace on the right-hand side is taken by means of eigenfunctions of $\hat{H}_{M}$, Eq. (38) is rewritten as

$$
\left\langle\hat{D}^{(1)}\left(\mathbf{r}_{1} \zeta_{1}, \mathbf{r}_{2} \zeta_{2}\right)\right\rangle_{H_{M}}=\frac{1}{\Xi} \sum_{m} e^{-\beta E_{m}}\left\langle\hat{D}^{(1)}\left(\mathbf{r}_{1} \zeta_{1}, \mathbf{r}_{2} \zeta_{2}\right)\right\rangle_{\Phi_{m}},
$$

where

$$
\hat{H}_{M}\left|\Phi_{m}\right\rangle=E_{m}\left|\Phi_{m}\right\rangle
$$

The RDM1 of the system of state $\left|\Phi_{m}\right\rangle$, i.e., $\left\langle\hat{D}^{(1)}\left(\mathbf{r}_{1} \zeta_{1}, \mathbf{r}_{2} \zeta_{2}\right)\right\rangle_{\Phi_{m}}$, can be rewritten by using the spectrum decomposition such that

$$
\left\langle\hat{D}^{(1)}\left(\mathbf{r}_{1} \zeta_{1}, \mathbf{r}_{2} \zeta_{2}\right)\right\rangle_{\Phi_{m}}=\sum_{\mu_{\Phi_{m}}} n_{\mu_{\Phi_{m}}}^{(1)} \mu_{\Phi_{m}}\left(\mathbf{r}_{1} \zeta_{1}\right) \mu_{\Phi_{m}}\left(\mathbf{r}_{2} \zeta_{2}\right)^{*}
$$

Substituting Eq. (41) into Eq. (39), we have

$$
\left\langle\hat{D}^{(1)}\left(\mathbf{r}_{1} \zeta_{1}, \mathbf{r}_{2} \zeta_{2}\right)\right\rangle_{H_{M}}=\frac{1}{\Xi} \sum_{m} e^{-\beta E_{m}} \sum_{\mu_{\Phi_{m}}} n_{\mu_{\Phi_{m}}}^{(1)} \mu_{\Phi_{m}}\left(\mathbf{r}_{1} \zeta_{1}\right) \mu_{\Phi_{m}}\left(\mathbf{r}_{2} \zeta_{2}\right)^{*} .
$$

This is the spectrum decomposition of the RDM1 at the finite temperature.

In order to consider the idempotent of the RDM1, we calculate the following product of the RDM1's:

$$
\begin{aligned}
& \int\left\langle\hat{D}^{(1)}\left(\mathbf{r}_{1} \zeta_{1}, \mathbf{r}_{2} \zeta_{2}\right)\right\rangle_{H_{M}}\left\langle\hat{D}^{(1)}\left(\mathbf{r}_{2} \zeta_{2}, \mathbf{r}_{1} \zeta_{1}\right)\right\rangle_{H_{M}} d^{3} r_{1} d^{3} r_{2} d \zeta_{1} d \zeta_{2}=\sum_{m} \sum_{m^{\prime}} \frac{e^{-\beta E_{m}}}{\Xi} \frac{e^{-\beta E_{m^{\prime}}}}{\Xi} \\
& \times \sum_{\mu_{\Phi_{m}}} \sum_{\mu_{\Phi_{m^{\prime}}}} n_{\mu_{\Phi_{m}}}^{(1)} n_{\mu_{\Phi_{m^{\prime}}}}^{(1)} \int \mu_{\Phi_{m}}\left(\mathbf{r}_{1} \zeta_{1}\right) \mu_{\Phi_{m}}\left(\mathbf{r}_{2} \zeta_{2}\right) \mu_{\Phi_{m^{\prime}}}\left(\mathbf{r}_{2} \zeta_{2}\right)^{*} \mu_{\Phi_{m^{\prime}}}\left(\mathbf{r}_{1} \zeta_{1}\right)^{*} d^{3} r_{1} d^{3} r_{2} d \zeta_{1} d \zeta_{2} .
\end{aligned}
$$

On the other hand, using Eq. (42), we have

$$
\int\left\langle\hat{D}^{(1)}\left(\mathbf{r}_{1} \zeta_{1}, \mathbf{r}_{1} \zeta_{1}\right)\right\rangle_{H_{M}} d^{3} r_{1} d \zeta_{1}=\sum_{m} \sum_{\mu_{\Phi_{m}}} \frac{e^{-\beta E_{m}}}{\Xi} n_{\mu_{\Phi_{m}}}^{(1)}
$$


where the normalization of the natural spin orbital is used [73]. Let us consider the magnitude of $e^{-\beta E_{m}} / \Xi$ which is contained on the right-hand sides of both Eqs. (43) and (44)). Suppose that the eigenvalues $E_{m}$ in Eq. (40) take positive values, and that the ground state is non-degenerate. Then, the magnitude of $e^{-\beta E_{m}} / \Xi$ for the ground state is larger than those for the excited states, and especially at low temperature, $e^{-\beta E_{m}} / \Xi$ would be nearly equal to unity and zero for the ground state and excited states, respectively. This speculation seems to be appropriate because there generally exists the energy gap between the ground state and excited states for superconductors. Of course it is rigorously correct at zero temperature. Further discussion will be presented in Sec. III-D.

Accordingly, the dominant contribution in Eq. (43) at low temperature comes from the case for $\left|\Phi_{m}\right\rangle=\left|\Phi_{m^{\prime}}\right\rangle=$ ground state. That is to say, Eq. (43) is approximated at low temperature in the following form:

$$
\int\left\langle\hat{D}^{(1)}\left(\mathbf{r}_{1} \zeta_{1}, \mathbf{r}_{2} \zeta_{2}\right)\right\rangle_{H_{M}}\left\langle\hat{D}^{(1)}\left(\mathbf{r}_{2} \zeta_{2}, \mathbf{r}_{1} \zeta_{1}\right)\right\rangle_{H_{M}} d^{3} r_{1} d^{3} r_{2} d \zeta_{1} d \zeta_{2} \approx \sum_{\mu_{\Phi_{m}}}\left(\frac{e^{-\beta E_{m}}}{\Xi} n_{\mu_{\Phi_{m}}}^{(1)}\right)^{2}
$$

where we use the orthonormality of the natural orbitals, and where the summation of the right-hand side is over the natural spin orbitals only for the ground state. Similarly, Eq. (44) is approximated at low temperature as

$$
\int\left\langle\hat{D}^{(1)}\left(\mathbf{r}_{1} \zeta_{1}, \mathbf{r}_{1} \zeta_{1}\right)\right\rangle_{H_{M}} d^{3} r_{1} d \zeta_{1} \approx \sum_{\mu_{\Phi_{m}}} \frac{e^{-\beta E_{m}}}{\Xi} n_{\mu_{\Phi_{m}}}^{(1)}
$$

where the note on the summation is the same as that of Eq. (45).

Furthermore, since the eigenfunction $\left|\Phi_{m}\right\rangle$ of Eq. (40) is the single Slater determinant in terms of the quasiparticle, and since the eigenvalue $n_{\mu_{\Phi_{m}}}^{(1)}$ corresponds to the occupation number of the natural spin orbital in the single Slater determinant $\left|\Phi_{m}\right\rangle[2]$, the value of $n_{\mu_{\Phi_{m}}}^{(1)}$ is necessarily equal to unity or zero. Thus, we have

$$
\frac{e^{-\beta E_{m}}}{\Xi} n_{\mu_{m}}^{(1)} \approx 1 \text { or } 0
$$

Subtracting Eq. (46) from Eq. (45) on both sides, we have

$$
\begin{aligned}
& \int\left\langle\hat{D}^{(1)}\left(\mathbf{r}_{1} \zeta_{1}, \mathbf{r}_{2} \zeta_{2}\right)\right\rangle_{H_{M}}\left\langle\hat{D}^{(1)}\left(\mathbf{r}_{2} \zeta_{2}, \mathbf{r}_{1} \zeta_{1}\right)\right\rangle_{H_{M}} d^{3} r_{1} d^{3} r_{2} d \zeta_{1} d \zeta_{2}-\int\left\langle\hat{D}^{(1)}\left(\mathbf{r}_{1} \zeta_{1}, \mathbf{r}_{1} \zeta_{1}\right)\right\rangle_{H_{M}} d^{3} r_{1} d \zeta_{1} \\
\approx & \sum_{\mu_{\Phi_{m}}}\left(\frac{e^{-\beta E_{m}}}{\Xi} n_{\mu_{\Phi_{m}}}^{(1)}\right)\left(\frac{e^{-\beta E_{m}}}{\Xi} n_{\mu_{\Phi_{m}}}^{(1)}-1\right) .
\end{aligned}
$$


Due to Eq. (47), the right-hand side of Eq. (48) is approximately equal to zero at low temperature. We finally obtain

$$
\int\left\langle\hat{D}^{(1)}\left(\mathbf{r}_{1} \zeta_{1}, \mathbf{r}_{2} \zeta_{2}\right)\right\rangle_{H_{M}}\left\langle\hat{D}^{(1)}\left(\mathbf{r}_{2} \zeta_{2}, \mathbf{r}_{1} \zeta_{1}\right)\right\rangle_{H_{M}} d^{3} r_{1} d^{3} r_{2} d \zeta_{1} d \zeta_{2} \approx \int\left\langle\hat{D}^{(1)}\left(\mathbf{r}_{1} \zeta_{1}, \mathbf{r}_{1} \zeta_{1}\right)\right\rangle_{H_{M}} d^{3} r_{1} d \zeta_{1}
$$

Thus, it is shown that the idempotent of the RDM1 approximately holds at low temperature even in the superconducting state. It should be noted that Eq. (49) rigorously holds at zero temperature.

\section{Fluctuation of the particle number for superconductors at low temperature}

In this section, it is shown that the fluctuation of the particle number is equal to $O(N)$ when the OPSS takes nonzero value.

Under the condition that $\mathbf{r}_{1}, \zeta_{1}=\mathbf{r}_{4}, \zeta_{4}$ and $\mathbf{r}_{2}, \zeta_{2}=\mathbf{r}_{3}, \zeta_{3}$, the Bloch-de Dominicis theorem for the RDM2, i.e., Eq. (35), is written as

$$
\begin{aligned}
\left\langle\psi^{\dagger}\left(\mathbf{r}_{1} \zeta_{1}\right) \psi^{\dagger}\left(\mathbf{r}_{2} \zeta_{2}\right) \psi\left(\mathbf{r}_{2} \zeta_{2}\right) \psi\left(\mathbf{r}_{1} \zeta_{1}\right)\right\rangle_{H_{M}} & =\left\langle\psi^{\dagger}\left(\mathbf{r}_{1} \zeta_{1}\right) \psi\left(\mathbf{r}_{1} \zeta_{1}\right)\right\rangle_{H_{M}}\left\langle\psi^{\dagger}\left(\mathbf{r}_{2} \zeta_{2}\right) \psi\left(\mathbf{r}_{2} \zeta_{2}\right)\right\rangle_{H_{M}} \\
& -\left\langle\psi^{\dagger}\left(\mathbf{r}_{1} \zeta_{1}\right) \psi\left(\mathbf{r}_{2} \zeta_{2}\right)\right\rangle_{H_{M}}\left\langle\psi^{\dagger}\left(\mathbf{r}_{2} \zeta_{2}\right) \psi\left(\mathbf{r}_{1} \zeta_{1}\right)\right\rangle_{H_{M}} \\
& +\left\langle\psi^{\dagger}\left(\mathbf{r}_{1} \zeta_{1}\right) \psi^{\dagger}\left(\mathbf{r}_{2} \zeta_{2}\right)\right\rangle_{H_{M}}\left\langle\psi\left(\mathbf{r}_{2} \zeta_{2}\right) \psi\left(\mathbf{r}_{1} \zeta_{1}\right)\right\rangle_{H_{M}}
\end{aligned}
$$

Using the anti-commutation relation of the field operators of electrons in the left-hand side, Eq. (50) is rewritten as

$$
\begin{aligned}
\left\langle\psi^{\dagger}\left(\mathbf{r}_{1} \zeta_{1}\right) \psi\left(\mathbf{r}_{1} \zeta_{1}\right) \psi^{\dagger}\left(\mathbf{r}_{2} \zeta_{2}\right) \psi\left(\mathbf{r}_{2} \zeta_{2}\right)\right\rangle_{H_{M}} & =\left\langle\psi^{\dagger}\left(\mathbf{r}_{1} \zeta_{1}\right) \psi\left(\mathbf{r}_{2} \zeta_{2}\right)\right\rangle_{H_{M}} \delta\left(\mathbf{r}_{1}-\mathbf{r}_{2}\right) \delta_{\zeta_{1} \zeta_{2}} \\
& +\left\langle\psi^{\dagger}\left(\mathbf{r}_{1} \zeta_{1}\right) \psi\left(\mathbf{r}_{1} \zeta_{1}\right)\right\rangle_{H_{M}}\left\langle\psi^{\dagger}\left(\mathbf{r}_{2} \zeta_{2}\right) \psi\left(\mathbf{r}_{2} \zeta_{2}\right)\right\rangle_{H_{M}} \\
& -\left\langle\psi^{\dagger}\left(\mathbf{r}_{1} \zeta_{1}\right) \psi\left(\mathbf{r}_{2} \zeta_{2}\right)\right\rangle_{H_{M}}\left\langle\psi^{\dagger}\left(\mathbf{r}_{2} \zeta_{2}\right) \psi\left(\mathbf{r}_{1} \zeta_{1}\right)\right\rangle_{H_{M}} \\
& +\left\langle\psi^{\dagger}\left(\mathbf{r}_{1} \zeta_{1}\right) \psi^{\dagger}\left(\mathbf{r}_{2} \zeta_{2}\right)\right\rangle_{H_{M}}\left\langle\psi\left(\mathbf{r}_{2} \zeta_{2}\right) \psi\left(\mathbf{r}_{1} \zeta_{1}\right)\right\rangle_{H_{M}} .
\end{aligned}
$$

Integrating both sides with respect to $\mathbf{r}_{1}, \mathbf{r}_{2}$ and $\zeta_{1}, \zeta_{2}$, we obtain

$$
\begin{aligned}
\left\langle\hat{N}^{2}\right\rangle_{H_{M}} & =\langle\hat{N}\rangle_{H_{M}}+\left(\langle\hat{N}\rangle_{H_{M}}\right)^{2} \\
& -\int\left\langle\hat{D}^{(1)}\left(\mathbf{r}_{1} \zeta_{1}, \mathbf{r}_{2} \zeta_{2}\right)\right\rangle_{H_{M}}\left\langle\hat{D}^{(1)}\left(\mathbf{r}_{2} \zeta_{2}, \mathbf{r}_{1} \zeta_{1}\right)\right\rangle_{H_{M}} d^{3} r_{1} d \zeta_{1} d^{3} r_{2} d \zeta_{2} \\
& +\int\left\langle\psi^{\dagger}\left(\mathbf{r}_{1} \zeta_{1}\right) \psi^{\dagger}\left(\mathbf{r}_{2} \zeta_{2}\right)\right\rangle_{H_{M}}\left\langle\psi\left(\mathbf{r}_{2} \zeta_{2}\right) \psi\left(\mathbf{r}_{1} \zeta_{1}\right)\right\rangle_{H_{M}} d^{3} r_{1} d \zeta_{1} d^{3} r_{2} d \zeta_{2},
\end{aligned}
$$


where Eqs. (36) and (39) are used, and where $\hat{N}$ is given by

$$
\hat{N}=\int \psi^{\dagger}\left(\mathbf{r}_{1} \zeta_{1}\right) \psi\left(\mathbf{r}_{1} \zeta_{1}\right) d^{3} r_{1} d \zeta_{1}
$$

Using Eqs. (49) and (53), the third term on the right-hand side of Eq. (52) can be approximated as

$$
\int\left\langle\hat{D}^{(1)}\left(\mathbf{r}_{1} \zeta_{1}, \mathbf{r}_{2} \zeta_{2}\right)\right\rangle_{H_{M}}\left\langle\hat{D}^{(1)}\left(\mathbf{r}_{2} \zeta_{2}, \mathbf{r}_{1} \zeta_{1}\right)\right\rangle_{H_{M}} d^{3} r_{1} d \zeta_{1} d^{3} r_{2} d \zeta_{2} \approx\langle\hat{N}\rangle_{H_{M}}
$$

at low temperature. Substituting Eq. (54) into Eq. (52), we finally get

$$
\left\langle\hat{N}^{2}\right\rangle_{H_{M}}-\left(\langle\hat{N}\rangle_{H_{M}}\right)^{2} \approx \int\left\langle\psi^{\dagger}\left(\mathbf{r}_{1} \zeta_{1}\right) \psi^{\dagger}\left(\mathbf{r}_{2} \zeta_{2}\right)\right\rangle_{H_{M}}\left\langle\psi\left(\mathbf{r}_{2} \zeta_{2}\right) \psi\left(\mathbf{r}_{1} \zeta_{1}\right)\right\rangle_{H_{M}} d^{3} r_{1} d \zeta_{1} d^{3} r_{2} d \zeta_{2}
$$

The physical quantity $\left\langle\psi^{\dagger}\left(\mathbf{r}_{1} \zeta_{1}\right) \psi^{\dagger}\left(\mathbf{r}_{2} \zeta_{2}\right)\right\rangle_{H_{M}}$ or $\left\langle\psi\left(\mathbf{r}_{2} \zeta_{2}\right) \psi\left(\mathbf{r}_{1} \zeta_{1}\right)\right\rangle_{H_{M}}$ is exactly the OPSS which has been mentioned in Sec. II-D. When the system is in the superconducting state, $\left\langle\psi\left(\mathbf{r}_{2} \zeta_{2}\right) \psi\left(\mathbf{r}_{1} \zeta_{1}\right)\right\rangle_{H_{M}}$ is given by Eq. (29), and Eq. (55) becomes

$$
\left\langle\hat{N}^{2}\right\rangle_{H_{M}}-\left(\langle\hat{N}\rangle_{H_{M}}\right)^{2} \approx 2 n_{\nu_{H_{M}}^{\max }}^{(2)} \int \nu_{H_{M}}^{\max }\left(\mathbf{r}_{1} \zeta_{1}, \mathbf{r}_{2} \zeta_{2}\right)^{*} \nu_{H_{M}}^{\max }\left(\mathbf{r}_{1} \zeta_{1}, \mathbf{r}_{2} \zeta_{2}\right) d^{3} r_{1} d \zeta_{1} d^{3} r_{2} d \zeta_{2}
$$

Using the normalization of $\left|\nu_{H_{M}}^{\max }\right\rangle$ and applying Eqs. (10) and (26), we finally get

$$
\left\langle\hat{N}^{2}\right\rangle_{H_{M}}-\left(\langle\hat{N}\rangle_{H_{M}}\right)^{2} \approx O(N)
$$

Thus, it is shown that the fluctuation of the particle number becomes $O(N)$ when the system is at low temperature in the superconducting state.

\section{Two kinds of fluctuations of the particle number in the superconducting state}

In the superconducting state, two kinds of fluctuations of the particle number can be generally observed. They are

(a) Statistical fluctuation of the particle number,

(b) Quantum fluctuation of the particle number.

The fluctuation (a) necessarily appears when the system is treated on the basis of the grand canonical ensemble [74]. This is because the fluctuation (a) originates from the fact that the 
system is in the statistically-mixed state at the finite temperature. As is well known [74], the magnitude of the fluctuation (a) is $O(N)$. In the limit of zero temperature, the system is close to the pure state, which results in the disappearance of the statistical fluctuation of the particle number. The fluctuation (a) vanishes in the limit of zero temperature. Above the critical temperature of the superconductivity, the fluctuation (a) does not disappear and would become larger because the probabilities of the occurrence of states possessing the different numbers of particles increase with temperature.

On the other hand, the fluctuation (b) appears only if the system is in the superconducting state, which has been shown in Sec. III-C. The fluctuation (b) originates from the spontaneous breaking of the gauge symmetry in the superconducting state, and does not from the statistically-mixed states. Therefore, the fluctuation (b) remains nonzero even at zero temperature, but vanishes at the critical temperature of the superconductivity. As the typical examples of this type of fluctuation, we can come up with the cases of the BCS ground state [65, 75] and the coherent state of the boson system [76]. In reference to the discussion on the exciton [77, 78], the latter case corresponds to the limiting case where the distance of particles forming the geminal is infinitely small and where the density of geminals is dilute. In both cases, the fluctuation of the particle number is shown to be $O(N)$ at zero temperature [75, 76, 79].

Here we shall give a comment on the discussion in Sec. III-C. Both fluctuations (a) and (b) should appear below the critical temperature. However, only the fluctuation (b) has been confirmed in Sec. III-C. This is not surprising because the fluctuation (a) becomes small at low temperature, and disappears in the limit of zero temperature. At low temperature, the fluctuation (a) can reasonably be disregarded compared to the fluctuation (b). In Sec. III-C, we have purposely focused on the case of the low temperature in order to evaluate the effects of the quantum fluctuation of the particle number.

The fluctuations (a) and (b) are expected to be observed together in the superconducting state. The fluctuation (a) is an increasing function of the temperature regardless of above or below the critical temperature. On the other hand, the fluctuation (b) is a decreasing function of the temperature, and becomes zero at the critical temperature. The temperature dependence of the fluctuation obtained from the combined (a) and (b) would have a gentle slope below the critical temperature, while it would have a steep slope above the critical temperature. Using this fact, the critical temperature can be estimated quantitatively as the 
major changing point of the slope in the above-mentioned curve. That is to say, it is possible to estimate the critical temperature of the superconductivity by means of the fluctuation of the particle number. This is a strong merit of the PD functional theory which will be presented in the subsequent section.

\section{PAIR-DENSITY FUNCTIONAL THEORY FOR SUPERCONDUCTORS}

In the previous sections, it is shown that the fluctuation of the particle number may become an indication to judge whether the superconducting state appears or not. In Sec. IV-A, we shall show that the fluctuation of the particle number can be calculated directly by means of the PD and electron density. Considering this fact, the first thing we should do is to develop the first-principles theory where the PD and electron density are quantitatively reproduced. In Sec. IV-B, we present the theoretical framework of the PD functional theory for superconductors.

\section{A. Relation between the fluctuation of the particle number and PD}

In this subsection, it is shown that the fluctuation of the particle number can be calculated directly via the PD and electron density. The PD is defined as the diagonal elements of the RDM2. Using Eq. (11), the operator of the PD, if it is denoted as $\hat{\gamma}^{(2)}\left(\mathbf{r}_{1} \zeta_{1}, \mathbf{r}_{2} \zeta_{2} ; \mathbf{r}_{1} \zeta_{1}, \mathbf{r}_{2} \zeta_{2}\right)$, is given by [32]

$$
\hat{\gamma}^{(2)}\left(\mathbf{r}_{1} \zeta_{1}, \mathbf{r}_{2} \zeta_{2} ; \mathbf{r}_{1} \zeta_{1}, \mathbf{r}_{2} \zeta_{2}\right)=\frac{1}{2} \psi^{\dagger}\left(\mathbf{r}_{1} \zeta_{1}\right) \psi^{\dagger}\left(\mathbf{r}_{2} \zeta_{2}\right) \psi\left(\mathbf{r}_{2} \zeta_{2}\right) \psi\left(\mathbf{r}_{1} \zeta_{1}\right) .
$$

Using Eqs. (53) and (58), the operator $\hat{N}^{2}$ is expressed using $\hat{\gamma}^{(2)}\left(\mathbf{r}_{1} \zeta_{1}, \mathbf{r}_{2} \zeta_{2} ; \mathbf{r}_{1} \zeta_{1}, \mathbf{r}_{2} \zeta_{2}\right)$ and $\hat{D}^{(1)}\left(\mathbf{r}_{1} \zeta_{1}, \mathbf{r}_{1} \zeta_{1}\right)$. We have

$$
\hat{N}^{2}=2 \iint \hat{\gamma}^{(2)}\left(\mathbf{r}_{1} \zeta_{1}, \mathbf{r}_{2} \zeta_{2} ; \mathbf{r}_{1} \zeta_{1}, \mathbf{r}_{2} \zeta_{2}\right) d^{3} r_{1} d^{3} r_{2} d \zeta_{1} d \zeta_{2}+\int \hat{D}^{(1)}(\mathbf{r} \zeta, \mathbf{r} \zeta) d^{3} r d \zeta,
$$

where note that $\hat{D}^{(1)}\left(\mathbf{r}_{1} \zeta_{1}, \mathbf{r}_{1} \zeta_{1}\right)$ is identical with the operator of the electron density. Therefore, the fluctuation of the particle number for the system with the statistical density matrix $\hat{\rho}_{H}$ is

$$
\begin{aligned}
\left\langle\hat{N}^{2}\right\rangle_{H}-\langle\hat{N}\rangle_{H}^{2} & =2 \iint\left\langle\hat{\gamma}^{(2)}\left(\mathbf{r}_{1} \zeta_{1}, \mathbf{r}_{2} \zeta_{2} ; \mathbf{r}_{1} \zeta_{1}, \mathbf{r}_{2} \zeta_{2}\right)\right\rangle_{H} d^{3} r_{1} d^{3} r_{2} d \zeta_{1} d \zeta_{2} \\
& +\int\left\langle\hat{D}^{(1)}(\mathbf{r} \zeta, \mathbf{r} \zeta)\right\rangle_{H} d^{3} r d \zeta-\left\{\int\left\langle\hat{D}^{(1)}(\mathbf{r} \zeta, \mathbf{r} \zeta)\right\rangle_{H} d^{3} r d \zeta\right\}^{2}
\end{aligned}
$$


The fluctuation of the particle number can be calculated rigorously by means of the PD and electron density through Eq. (60). In order to predict the fluctuation of the particle number, that is to say, in order to observe whether the OPSS appears in the system or not, we may just develop the first-principles theory in which both the PD and electron density are reproduced.

It should be noted that the electron density can be derived from the PD when the system is in the normal state [61], while it cannot be done in the superconducting state. There is no connection between the PD and electron density in the superconducting state. This is because the superconducting state does not satisfy the particle number conservation [80]. In the superconducting state, we have to choose both the PD and electron density as the basic variables for describing the fluctuation of the particle number.

\section{B. Pair-density functional theory}

In order to reproduce the fluctuation of the particle number, the PD functional theory seems to be most suitable because that for the normal state has been developed so far by many workers [30 60], and because the findings and knowledge obtained by them may be useful for developing the theory for superconductors.

In a similar way to the zero-temperature PD functional theory [32 40$]$, the finite temperature PD functional theory can be formulated on the basis of so-called the "Hohenberg-Kohn theorem". It is composed of two theorems; one is the variational principle with respect to the PD and electron density, and another is the theorem concerning one-to-one correspondence between the correct density matrix and equilibrium densities. We shall give the proofs of them below.

Let us start with the Hamiltonian for superconductors. It includes the kinetic energy $\hat{T}$, electron-electron repulsive potential energy $\hat{W}_{1}$, external potential energy $\hat{V}$, and electronelectron attractive potential energy $\hat{W}_{2}$ which is mediated by the quasiparticle such as a phonon. We have

$$
\hat{H}=\hat{T}+\hat{W}_{1}+\hat{W}_{2}+\hat{V},
$$

with

$$
\hat{T}=\sum_{\sigma} \int \psi_{\sigma}^{\dagger}(\mathbf{r}) \frac{\mathbf{p}^{2}}{2 m} \psi_{\sigma}(\mathbf{r}) d^{3} r
$$




$$
\begin{aligned}
& \hat{W}_{1}=\frac{1}{2} \sum_{\sigma} \sum_{\sigma^{\prime}} \iint \psi_{\sigma}^{\dagger}(\mathbf{r}) \psi_{\sigma^{\prime}}^{\dagger}\left(\mathbf{r}^{\prime}\right) \frac{e^{2}}{\left|\mathbf{r}-\mathbf{r}^{\prime}\right|} \psi_{\sigma^{\prime}}\left(\mathbf{r}^{\prime}\right) \psi_{\sigma}(\mathbf{r}) d^{3} r d^{3} r^{\prime} \\
& \hat{W}_{2}=\frac{1}{2} \sum_{\sigma} \sum_{\sigma^{\prime}} \iint \psi_{\sigma}^{\dagger}(\mathbf{r}) \psi_{\sigma^{\prime}}^{\dagger}\left(\mathbf{r}^{\prime}\right) w\left(\mathbf{r}, \mathbf{r}^{\prime}\right) \psi_{\sigma^{\prime}}\left(\mathbf{r}^{\prime}\right) \psi_{\sigma}(\mathbf{r}) d^{3} r d^{3} r^{\prime}, \\
& \hat{V}=\sum_{\sigma} \int v(\mathbf{r}) \psi_{\sigma}^{\dagger}(\mathbf{r}) \psi_{\sigma}(\mathbf{r}) d^{3} r
\end{aligned}
$$

where $\psi_{\sigma}(\mathbf{r})$ is the field operator of the electron with spin $\sigma$, and where $w\left(\mathbf{r}, \mathbf{r}^{\prime}\right)$ and $v(\mathbf{r})$ are the attractive potential and external potential, respectively.

We construct the PD functional theory in accordance with the way of the extended constrained-search theory $\left[\begin{array}{ll}81 & 85\end{array}\right][25]$. The PD and electron density are chosen as the basic variables which determine the properties of the equilibrium state of the system. The statistical averages of them with respect to the statistical density matrix $\hat{\rho}$ are, respectively, written as

$$
\begin{aligned}
& \gamma^{(2)}\left(\mathbf{r} \zeta, \mathbf{r}^{\prime} \zeta^{\prime} ; \mathbf{r} \zeta, \mathbf{r}^{\prime} \zeta^{\prime}\right) \\
= & \operatorname{Tr}\left\{\hat{\rho} \hat{\gamma}^{(2)}\left(\mathbf{r} \zeta, \mathbf{r}^{\prime} \zeta^{\prime} ; \mathbf{r} \zeta, \mathbf{r}^{\prime} \zeta^{\prime}\right)\right\} \\
= & \frac{1}{2} \sum_{\sigma_{1}} \sum_{\sigma_{2}} \sum_{\sigma_{3}} \sum_{\sigma_{4}} \operatorname{Tr}\left\{\hat{\rho} \psi_{\sigma_{1}}^{\dagger}(\mathbf{r}) \psi_{\sigma_{2}}^{\dagger}\left(\mathbf{r}^{\prime}\right) \psi_{\sigma_{3}}\left(\mathbf{r}^{\prime}\right) \psi_{\sigma_{4}}(\mathbf{r})\right\} \chi_{\sigma_{1}}(\zeta) \chi_{\sigma_{4}}(\zeta) \chi_{\sigma_{2}}\left(\zeta^{\prime}\right) \chi_{\sigma_{3}}\left(\zeta^{\prime}\right),
\end{aligned}
$$

and

$$
\begin{aligned}
n(\mathbf{r} \zeta) & =\operatorname{Tr}\{\hat{\rho} \hat{n}(\mathbf{r} \zeta)\} \\
& =\sum_{\sigma_{1}} \sum_{\sigma_{2}} \operatorname{Tr}\left\{\hat{\rho} \psi_{\sigma_{1}}^{\dagger}(\mathbf{r}) \psi_{\sigma_{2}}(\mathbf{r})\right\} \chi_{\sigma_{1}}(\zeta) \chi_{\sigma_{2}}(\zeta)
\end{aligned}
$$

Here we define the universal energy functional that are independent of the external field:

$$
F\left[\gamma^{(2)}, n\right]=\operatorname{Min}_{\hat{\rho} \rightarrow\left(\gamma^{(2)}, n\right)} \operatorname{Tr}\left\{\hat{\rho}\left(\hat{T}+\hat{W}_{1}+\hat{W}_{2}\right)+\frac{1}{\beta} \hat{\rho} \ln \hat{\rho}\right\},
$$

where the right-hand side means that the minimum value of the statistical average of $\hat{T}+$ $\hat{W}_{1}+\hat{W}_{2}$ plus entropy-related term is searched by varying statistical density matrices within the set of those that yield prescribed $\gamma^{(2)}$ and $n$. The last term in the curly bracket on the right-hand side is the entropy term, the operator of which is given by $\hat{S}=-k_{B} \hat{\rho} \ln \hat{\rho}$.

We shall discuss the Hohenberg-Kohn theorem for the present PD functional theory. With the use of Eq. (68), the variational principle with respect to the PD and electron density can be derived by rewriting Gibbs's variational principle for the equilibrium density 
matrix [86]. First the grand-potential functional in terms of the density matrix is defined as follows:

$$
\Omega[\hat{\rho}]=\operatorname{Tr}\left\{\hat{\rho}(\hat{H}-\mu \hat{N})+\frac{1}{\beta} \hat{\rho} \ln \hat{\rho}\right\},
$$

where $\hat{N}$ is the operator of the particle number which is given by $\hat{N}=\int \hat{n}(\mathbf{r}, \zeta) d^{3} r d \zeta$. Gibbs's variational principle says that the above functional $\Omega[\hat{\rho}]$ takes the minimum value at the correct density matrix $\hat{\rho}_{0}$ and the corresponding value $\Omega\left[\hat{\rho}_{0}\right]$ is equal to the correct grand potential, i.e., the grand potential of the equilibrium state $\Omega_{0}$. Namely we have

$$
\Omega_{0}=\operatorname{Min}_{\hat{\rho}} \Omega[\hat{\rho}]=\Omega\left[\hat{\rho}_{0}\right]
$$

where $\hat{\rho}_{0}$ is given by [86]

$$
\hat{\rho}_{0}=\frac{e^{-\beta(\hat{H}-\mu \hat{N})}}{\Xi},
$$

with $\Xi=\operatorname{Tr}\left\{e^{-\beta(\hat{H}-\mu \hat{N})}\right\}$. Equation (170) is formally divided into two-step variations such that

$$
\begin{aligned}
\Omega_{0} & =\operatorname{Min}_{\gamma^{(2)}, n}\left\{\underset{\hat{\rho} \rightarrow\left(\gamma^{(2)}, n\right)}{\operatorname{Min}_{[\hat{\rho}]\}}}\right. \\
& =\operatorname{Min}_{\gamma^{(2)}, n}\left[\underset{\hat{\rho} \rightarrow\left(\gamma^{(2)}, n\right)}{\operatorname{Min}}\left[\operatorname{Tr}\left\{\hat{\rho}\left(\hat{T}+\hat{W}_{1}+\hat{W}_{2}\right)+\frac{1}{\beta} \hat{\rho} \ln \hat{\rho}\right\}+\operatorname{Tr}\{\hat{\rho}(\hat{V}-\mu \hat{N})\}\right]\right] \\
& =\operatorname{Min}_{\gamma^{(2)}, n}\left[F\left[\gamma^{(2)}, n\right]+\int\{v(\mathbf{r})-\mu\} n(\mathbf{r}, \zeta) d^{3} r d \zeta\right]
\end{aligned}
$$

where Eqs. (65), (67) and (68) are used. If we define the grand potential functional which is given by

$$
\Omega_{v-\mu}\left[\gamma^{(2)}, n\right]=F\left[\gamma^{(2)}, n\right]+\int\{v(\mathbf{r})-\mu\} n(\mathbf{r}, \zeta) d^{3} r d \zeta
$$

then Eq. (72) is rewritten as

$$
\Omega_{0}=\underset{\gamma^{(2)}, n}{\operatorname{Min}} \Omega_{v-\mu}\left[\gamma^{(2)}, n\right]
$$

Compared the first line of the right-hand side of Eq. (172) with the third line, the functional $\Omega_{v-\mu}\left[\gamma^{(2)}, n\right]$ is written as

$$
\Omega_{v-\mu}\left[\gamma^{(2)}, n\right]=\operatorname{Min}_{\hat{\rho} \rightarrow\left(\gamma^{(2)}, n\right)} \Omega[\hat{\rho}]
$$

The value of $\Omega_{v-\mu}\left[\gamma^{(2)}, n\right]$ corresponds to the grand potential at the minimum point within the restricted set of density matrices that yield the prescribed $\gamma^{(2)}$ and $n$. Therefore, Eq. 
(74) means that the global minimum point is searched within the set of local minimum points searched by Eq. (75). Since such a global minimum point gives the correct grand potential $\Omega_{0}$, and using Eq. (70), the PD and electron density that are found via Eq. (74) corresponds to those that are calculated from the correct density matrix $\hat{\rho}_{0}$. They are exactly the PD and electron density for the equilibrium state of the system, which are hereafter denoted as $\gamma_{0}^{(2)}$ and $n_{0}$. Thus, Eq. (174) represents the variational principle with respect to the PD and electron density. The results are summarized as follows:

$$
\Omega_{0}=\underset{\gamma^{(2)}, n}{\operatorname{Min}} \Omega_{v-\mu}\left[\gamma^{(2)}, n\right]=\Omega_{v-\mu}\left[\gamma_{0}^{(2)}, n_{0}\right]
$$

with

$$
\begin{aligned}
& \gamma_{0}^{(2)}\left(\mathbf{r} \zeta, \mathbf{r}^{\prime} \zeta^{\prime} ; \mathbf{r} \zeta, \mathbf{r}^{\prime} \zeta^{\prime}\right)=\operatorname{Tr}\left\{\hat{\rho}_{0} \hat{\gamma}^{(2)}\left(\mathbf{r} \zeta, \mathbf{r}^{\prime} \zeta^{\prime} ; \mathbf{r} \zeta, \mathbf{r}^{\prime} \zeta^{\prime}\right)\right\} \\
& n_{0}(\mathbf{r} \zeta)=\operatorname{Tr}\left\{\hat{\rho}_{0} \hat{n}(\mathbf{r} \zeta)\right\} .
\end{aligned}
$$

Next we discuss the theorem for one-to-one correspondence between the correct density matrix and equilibrium densities. The universal energy functional at the equilibrium densities is given by

$$
\begin{aligned}
& F\left[\gamma_{0}^{(2)}, n_{0}\right]=\operatorname{Min}_{\hat{\rho} \rightarrow\left(\gamma_{0}^{(2)}, n_{0}\right)} \operatorname{Tr}\left\{\hat{\rho}\left(\hat{T}+\hat{W}_{1}+\hat{W}_{2}\right)+\frac{1}{\beta} \hat{\rho} \ln \hat{\rho}\right\} \\
& =\operatorname{Tr}\left\{\hat{\rho}_{\text {min }}\left(\hat{T}+\hat{W}_{1}+\hat{W}_{2}\right)+\frac{1}{\beta} \hat{\rho}_{\text {min }} \ln \hat{\rho}_{\text {min }}\right\},
\end{aligned}
$$

where the searched density matrix of the right-hand side is referred to as $\hat{\rho}_{\text {min }}$, and where note that $\hat{\rho}_{\text {min }}$ yields $\gamma_{0}^{(2)}$ and $n_{0}$. Considering Eq. (70), the following relation holds:

$$
\begin{aligned}
& \operatorname{Tr}\left\{\hat{\rho}_{0}\left(\hat{T}+\hat{W}_{1}+\hat{W}_{2}+\hat{V}-\mu \hat{N}\right)+\frac{1}{\beta} \hat{\rho}_{0} \ln \hat{\rho}_{0}\right\} \\
& \leq \operatorname{Tr}\left\{\hat{\rho}_{\min }\left(\hat{T}+\hat{W}_{1}+\hat{W}_{2}+\hat{V}-\mu \hat{N}\right)+\frac{1}{\beta} \hat{\rho}_{\min } \ln \hat{\rho}_{\min }\right\} .
\end{aligned}
$$

Since both $\hat{\rho}_{0}$ and $\hat{\rho}_{\text {min }}$ yield the correct densities $\gamma_{0}^{(2)}$ and $n_{0}$, it follows that

$$
\operatorname{Tr}\left\{\hat{\rho}_{0}(\hat{V}-\mu \hat{N})\right\}=\operatorname{Tr}\left\{\hat{\rho}_{\min }(\hat{V}-\mu \hat{N})\right\}=\int\{v(\mathbf{r})-\mu\} n_{0}(\mathbf{r}, \zeta) d^{3} r d \zeta .
$$

Substitution of Eq. (80) into Eq. (79) leads to

$$
\begin{aligned}
& \operatorname{Tr}\left\{\hat{\rho}_{0}\left(\hat{T}+\hat{W}_{1}+\hat{W}_{2}\right)+\frac{1}{\beta} \hat{\rho}_{0} \ln \hat{\rho}_{0}\right\} \\
& \leq \operatorname{Tr}\left\{\hat{\rho}_{\text {min }}\left(\hat{T}+\hat{W}_{1}+\hat{W}_{2}\right)+\frac{1}{\beta} \hat{\rho}_{\text {min }} \ln \hat{\rho}_{\text {min }}\right\} .
\end{aligned}
$$


The right-hand side is coincident with $F\left[\gamma_{0}^{(2)}, n_{0}\right]$ from Eq. (78), which deduces that only an equal sign is satisfied in Eq. (81). Namely we have

$$
\begin{aligned}
& \operatorname{Tr}\left\{\hat{\rho}_{0}\left(\hat{T}+\hat{W}_{1}+\hat{W}_{2}\right)+\frac{1}{\beta} \hat{\rho}_{0} \ln \hat{\rho}_{0}\right\} \\
& =\operatorname{Tr}\left\{\hat{\rho}_{\text {min }}\left(\hat{T}+\hat{W}_{1}+\hat{W}_{2}\right)+\frac{1}{\beta} \hat{\rho}_{\text {min }} \ln \hat{\rho}_{\text {min }}\right\} .
\end{aligned}
$$

Using Eqs. (80) and (82), only an equal sign holds also in Eq. (79). The left-hand side of Eq. (79) is exactly the correct grand potential $\Omega_{0}$. Considering Gibbs's variational theorem (70), we finally get

$$
\hat{\rho}_{\min }=\hat{\rho}_{0}
$$

It follows that the correct density matrix $\hat{\rho}_{0}\left(=\hat{\rho}_{\text {min }}\right)$ is uniquely determined by the correct densities $\gamma_{0}^{(2)}$ and $n_{0}$ via Eq. (178), and vice versa due to Eq. (777). Thus, it is proved that one-to-one correspondence between the density matrix $\hat{\rho}_{0}$ and densities $\gamma_{0}^{(2)}, n_{0}$.

The most crucial theorems of the PD functional theory have successfully been derived. These theorems enable us to develop the concrete scheme for calculating the PD and electron density of the equilibrium state. This would be the next issue to be tackled.

\section{Discussions}

As mentioned in Sec. III, the fluctuation of the particle number is a possible indication of the superconducting state, and accordingly the critical temperature of the superconductivity may be evaluated via the temperature dependence of such a fluctuation. This seems to be one of the strong merits of the PD functional theory presented in the previous section.

In addition to the estimation of the critical temperature, the present theory has another merit about the description of the superconducting state. As shown below, it can predict the density of particles which form the geminal $\left|\nu_{H_{M}}^{\max }\right\rangle$. In other words, the spatial distribution of the OPSS can be obtained from the present PD functional theory.

As shown in Sec. III-B, the idempotent of the RDM1 holds at low temperature. This idempotent can also be written in the other form:

$$
\int\left\langle\hat{D}^{(1)}\left(\mathbf{r}_{1} \zeta_{1}, \mathbf{r}_{2} \zeta_{2}\right)\right\rangle_{H_{M}}\left\langle\hat{D}^{(1)}\left(\mathbf{r}_{2} \zeta_{2}, \mathbf{r}_{1} \zeta_{1}\right)\right\rangle_{H_{M}} d^{3} r_{2} d \zeta_{2} \approx\left\langle\hat{D}^{(1)}\left(\mathbf{r}_{1} \zeta_{1}, \mathbf{r}_{1} \zeta_{1}\right)\right\rangle_{H_{M}} .
$$


Integrating both sides of Eq. (50) with respect to $\mathbf{r}_{2}$ and $\zeta_{2}$, we have

$$
\begin{aligned}
& \int\left\langle\psi^{\dagger}\left(\mathbf{r}_{1} \zeta_{1}\right) \psi^{\dagger}\left(\mathbf{r}_{2} \zeta_{2}\right) \psi\left(\mathbf{r}_{2} \zeta_{2}\right) \psi\left(\mathbf{r}_{1} \zeta_{1}\right)\right\rangle_{H_{M}} d^{3} r_{2} d \zeta_{2} \\
= & \left\langle\psi^{\dagger}\left(\mathbf{r}_{1} \zeta_{1}\right) \psi\left(\mathbf{r}_{1} \zeta_{1}\right)\right\rangle_{H_{M}} \int\left\langle\psi^{\dagger}\left(\mathbf{r}_{2} \zeta_{2}\right) \psi\left(\mathbf{r}_{2} \zeta_{2}\right)\right\rangle_{H_{M}} d^{3} r_{2} d \zeta_{2} \\
- & \int\left\langle\psi^{\dagger}\left(\mathbf{r}_{1} \zeta_{1}\right) \psi\left(\mathbf{r}_{2} \zeta_{2}\right)\right\rangle_{H_{M}}\left\langle\psi^{\dagger}\left(\mathbf{r}_{2} \zeta_{2}\right) \psi\left(\mathbf{r}_{1} \zeta_{1}\right)\right\rangle_{H_{M}} d^{3} r_{2} d \zeta_{2} \\
+ & \int\left\langle\psi^{\dagger}\left(\mathbf{r}_{1} \zeta_{1}\right) \psi^{\dagger}\left(\mathbf{r}_{2} \zeta_{2}\right)\right\rangle_{H_{M}}\left\langle\psi\left(\mathbf{r}_{2} \zeta_{2}\right) \psi\left(\mathbf{r}_{1} \zeta_{1}\right)\right\rangle_{H_{M}} d^{3} r_{2} d \zeta_{2}
\end{aligned}
$$

Substituting Eq. (84) into Eq. (85), and using Eqs. (23), (29) and (39), Eq. (85) is rewritten as

$$
\begin{aligned}
& 2 \int\left\langle\hat{\gamma}^{(2)}\left(\mathbf{r}_{1} \zeta_{1} \mathbf{r}_{2} \zeta_{2} ; \mathbf{r}_{1} \zeta_{1} \mathbf{r}_{2} \zeta_{2}\right)\right\rangle_{H_{M}} d^{3} r_{2} d \zeta_{2}-\left\{\langle\hat{N}\rangle_{H_{M}}-1\right\}\left\langle\hat{n}\left(\mathbf{r}_{1} \zeta_{1}\right)\right\rangle_{H_{M}} \\
= & 2 n_{\nu^{\max }}^{(2)} \int \nu^{\max }\left(\mathbf{r}_{2} \zeta_{2} \mathbf{r}_{1} \zeta_{1}\right)^{*} \nu^{\max }\left(\mathbf{r}_{1} \zeta_{1} \mathbf{r}_{2} \zeta_{2}\right) d^{3} r_{2} d \zeta_{2} .
\end{aligned}
$$

The integral of the right-hand side represents the density of particles forming geminals in the superconducting state. Thus, the spatial distribution of the OPSS can be obtained by means of the PD and electron density at low temperature. This is also a useful information for understanding the superconducting state as well as the critical temperature.

\section{CONCLUDING REMARKS}

In this paper, we show that the fluctuation of the particle number is a possible indication of whether the superconducting state appears or not in the system. Specifically, the quantum fluctuation of the particle number becomes $O(N)$ when the OPSS appears in the system. Using this result, the critical temperature of the superconductivity can be evaluated in principle as a bending point in the temperature-dependence curve of the fluctuation of the particle number.

As the practical scheme for calculating the fluctuation of the particle number, we also present the theoretical framework of the finite-temperature PD functional theory. This PD functional theory can evaluate not only the fluctuation of the particle number but also the density of particles forming geminals of the BEC. The latter corresponds to the spatial distribution of the OPSS, which also characterizes the superconducting state of the system as well as the critical temperature. 
Let us discuss the meanings of the present PD functional theory from the viewpoint of the basic variables describing the superconducting state. In this paper, we present the theory where the fluctuation of the particle number, i.e., diagonal elements of the RDM2, are chosen as the basic variables, instead of choosing the off-diagonal elements of the RDM2 which have been used in the conventional theories for superconductors [9, 10, 25]. In the superconducting state, the fluctuation of the particle number is closely related to the OPSS, which is shown in Eq. (55). This means that the diagonal and off-diagonal elements of the RDM2 are related to each other in the superconducting state. Since the RDM2 is dependent on the superconducting state as shown in Eqs. (22) and (23), the diagonal and off-diagonal elements of the RDM2 are correlated mutually via the superconducting state. This is what we find in the present work. Although only the off-diagonal elements of the RDM2 have previously been adopted as the basic variables, the indication of the superconducting state necessarily appears also in the diagonal elements of the RDM2. This is exactly the quantum fluctuation of the particle number, which is the basic variable chosen in the present PD functional theory.

The next step is to construct the concrete scheme for calculating the PD and electron density on the basis of theorems of the present PD functional theory, and then to confirm the reproducibility of them through actual calculations. This will be done in near future.

\section{Acknowledgments}

This work was partially supported by Grant-in-Aid for Scientific Research (No. 26400354 and No. 26400397) of Japan Society for the Promotion of Science.

[1] C. N. Yang, Rev. Mod. Phys. 34, 694 (1962).

[2] A. J. Leggett, Quantum Liquids (Oxford University Press, Oxford, 2006).

[3] M. Ueda, Fundamentals and New Frontiers of Bose-Einstein Condensation (World Scientific, New Jersey, 2010).

[4] C. J. Pethick and H. Smith, Bose-Einstein Condensation in Dilute Gases (Cambridge University Press, Cambridge, 2008). 
[5] The order parameter of the superconducting state is reviewed in Sec. II together with the discussion on the properties of two-particle states which forms the BEC.

[6] The representative first-principles theories for superconductors are the Bogoliubov-de Gennes theory [7, 8] and density functional theory [9, 10]. The explanation of the latter is followed in the text.

[7] N. N. Bogoliubov, Uspekhi Fiz. Nauk., 67, 549 (1959).

[8] P. G. de Gennes, Superconductivity of Metals and Alloys (W. A. Benjamin, New York, 1966) Chap. 5.

[9] L. N. Oliveira, E. K. U. Gross and W. Kohn, Phys. Rev. Lett 60, 2430 (1988).

[10] W. Kohn, E. K. U. Gross and L. N. Oliveira, J. de Phyique (Paris) 50, 2601 (1989).

[11] M. Lüders, M. A. L. Marques, N. N. Lathiotakis, A. Floris, G. Profeta, L. Fast, A. Continenza, S. Massidda, and E. K. U. Gross, Phys. Rev. B 72, 024545 (2005).

[12] M. A. L. Marques, M. Lüders, N. N. Lathiotakis, G. Profeta, A. Floris, L. Fast, A. Continenza, E. K. U. Gross, and S. Massidda, Phys. Rev. B 72, 024546 (2005).

[13] T. Kreibich and E. K. U. Gross, Phys. Rev. Lett. 86, 2984 (2001).

[14] A. Floris, G. Profeta, N. N. Lathiotakis, M. Lüders, M. A. L. Marques, C. Franchini, E. K. U. Gross, A. Continenza, and S. Massidda, Phys. Rev. Lett. 94, 037004 (2005).

[15] G. Profeta, C. Franchini, N. N. Lathiotakis, A. Floris, A. Sanna, M. A. L. Marques, M. Lüders, S. Massidda, E. K. U. Gross, and A. Continenza, Phys. Rev. Lett. 96, 047003 (2006).

[16] A. Sanna, C. Franchini, A. Floris, G. Profeta, N. N. Lathiotakis, M. Lüders, M. A. L. Marques, E. K. U. Gross, A. Continenza, and S. Massidda, Phys. Rev. B 73, 144512 (2006).

[17] A. Floris, A. Sanna, S. Massidda, and E. K. U. Gross, Phys. Rev. B 75, 054508 (2007).

[18] A. Sanna, G. Profeta, A. Floris, A. Marini, E. K. U. Gross, and S. Massidda, Phys. Rev. B 75, 020511(R) (2007).

[19] P. Cudazzo, G. Profeta, A. Sanna, A. Floris, A. Continenza, S. Massidda, and E.K.U. Gross, Phys. Rev. Lett. 100, 257001 (2008); Phys. Rev. B 81, 134506 (2010).

[20] J. Quintanilla, K. Capelle, and L. N. Oliveira, Phys. Rev. B 78, 205426 (2008).

[21] C. Bersier, A. Floris, A. Sanna, G. Profeta, A. Continenza, E. K. U. Gross, and S. Massidda, Phys. Rev. B 79, 104503 (2009).

[22] G. Stefanucci, E. Perfetto, and M. Cini, Phys. Rev. B 81, 115446 (2010).

[23] R. Akashi, K. Nakamura, R. Arita, and M. Imada, Phys. Rev. B 86, 054513 (2012). 
[24] R. Akashi and R. Arita, Phys. Rev. Lett. 111, 057006 (2013); Phys. Rev. B 88, 014514 (2013).

[25] K. Higuchi, K. Koide, T. Imanishi, and M. Higuchi, Int. J. Quantum Chem. 113, 709 (2013).

[26] F. Essenberger, A. Sanna, A. Linscheid, F. Tandetzky, G. Profeta, P. Cudazzo, and E. K. U. Gross, Phys. Rev. B 90, 214504 (2014).

[27] G. Csire, B. Újfalussy, J. Cserti, and B. Győrffy, Phys. Rev. B 91, 165142 (2015).

[28] A. Linscheid, A. Sanna, F. Essenberger, and E. K. U. Gross, Phys. Rev. B 92, 024505 (2015).

[29] A. Linscheid, A. Sanna, A. Floris, and E. K. U. Gross, Phys. Rev. Lett. 115, 097002 (2015).

[30] P. Ziesche, Phys. Lett. A 195, 213 (1994).

[31] P. Ziesche, Int. J. Quantum Chem. 60, 1361 (1996).

[32] M. Higuchi and K. Higuchi, Physica B 387, 117 (2007).

[33] M. Higuchi and K. Higuchi, Phys. Rev. A 75, 042510 (2007).

[34] M. Higuchi and K. Higuchi, Phys. Rev. B 78, 125101 (2008).

[35] K. Higuchi and M. Higuchi, J. Phys.: Condens. Matter 21, 064206 (2009).

[36] K. Higuchi and M. Higuchi, Phys. Rev. B. 82, 155135 (2010).

[37] M. Higuchi and K. Higuchi, Phys. Rev. A 84, 044502 (2011).

[38] K. Higuchi and M. Higuchi, Phys. Rev. A 85, 062508 (2012).

[39] K. Higuchi and M. Higuchi, Phys. Rev. A 87, 032511 (2013).

[40] K. Higuchi and M. Higuchi, Phys. Rev. A. 90, 062511 (2014).

[41] A. Gonis, T. C. Schulthess, J. van Ek, and P. E. A. Turchi, Phys. Rev. Lett. 77, 2981 (1996).

[42] A. Gonis, T. C. Schulthess, P. E. A. Turchi, and J. van Ek, Phys. Rev. B 56, 9335 (1997).

[43] S. Kh. Samvelyan, Int. J. Quantum Chem. 65, 127 (1997).

[44] M. Levy and P. Ziesche, J. Chem. Phys. 115, 9110 (2001).

[45] Á. Nagy, Phys. Rev. A 66, 022505 (2002); Int. J. Quantum Chem. 106, 1043 (2006); Phys. Rev. A 90, 022505 (2014).

[46] Á. Nagy and C. Amovilli, J. Chem. Phys. 121, 6640 (2004); Chem. Phys. Lett. 469, 353 (2009); Phys. Rev. A 82, 042510 (2010).

[47] F. Furche, Phys. Rev. A 70, 022514 (2004).

[48] M.-E. Pistol, Chem. Phys. Lett. 400, 548 (2004); ibid. 417, 521 (2006); ibid. 422, 363 (2006); ibid. 431, 216 (2006); ibid. 449, 208 (2007).

[49] B. Hetényi, L. Brualla, and S. Fantoni, Phys. Rev. Lett. 93, 170202 (2004).

[50] J. K. Percus, J. Chem. Phys. 122, 234103 (2005). 
[51] P. W. Ayers and M. Levy, J. Chem. Sci. 117, 507 (2005).

[52] P. W. Ayers, J. Math. Phys. 46, 062107 (2005); Phys. Rev. A 74, 042502 (2006).

[53] P. W. Ayers, S. Golden, and M. Levy, J. Chem. Phys. 124, 054101 (2006).

[54] P. W. Ayers and E. R. Davidson, Int. J. Quantum Chem. 106, 1487 (2006).

[55] P. Gori-Giorgi and A. Savin, Philos. Mag. 86, 2643 (2006).

[56] P. W. Ayers and S. Liu, Phys. Rev. A 75, 022514 (2007).

[57] C. Amovilli and Á. Nagy, J. Chem. Phys. 129204108 (2008).

[58] R. Cuevas-Saavedra and P. W. Ayers, Int. J. Quantum Chem. 109, 1699 (2009).

[59] D. Chakraborty and P. W. Ayers, J. Math. Chem. 49, 1810 (2011); ibid. 49, 1822 (2011).

[60] N. Otero and M. Mandado, J. Comput. Chem. 33, 1240 (2012).

[61] R. G. Parr and W. Yang, Density-Functional Theory of Atoms and Molecules (Oxford University Press, New York, 1989) Chap. 2.

[62] This assumption is reasonable, because the superconductors usually have one kind of order parameters.

[63] It seems that electron pairings may lower the total energy of the system as can be seen in the BCS theory [64, 65]. If another kind of geminals belonging to type (b) exists, the occupation number of this geminal also becomes $O(N)$.

[64] L. N. Cooper, Phys. Rev. 104, 1189 (1956).

[65] J. Bardeen, L. N. Cooper and J. R. Schrieffer, Phys. Rev. 108, 1175 (1957).

[66] See, for example, E. T. Whittaker and G. N. Watson, A Course of Modern Analysis (Cambridge University Press, New York, 1927) Chap. 9.

[67] S. Weinberg, The Quantum Theory of Fields vol. 1 (Cambridge University Press, New York, 1995) Chap. 4.

[68] N. N. Bogoliubov, Nuovo Cimento 7, 794 (1958).

[69] J. G. Valatin, Nuovo Cimento 7, 843 (1958).

[70] C. Bloch and C. De Dominicis, Nucl. Phys. 7, 459 (1958).

[71] M. Gaudin, Nucl. Phys. 15, 89 (1960).

[72] The Bloch-de Dominicis theorem is sometimes called Wick's theorem at the finite temperature.

[73] The eigenfunction for the RDM1 is called the natural spin orbital in the field of the quantum chemistry [61].

[74] See, for example, W. Greiner, Thermodynamics and Statistical Mechanics (Springer-Verlag, 
New York, 1995) Chap. 9.

[75] See, for example, M. Tinkham, Introduction to Superconductivity (Dover Pub., Mew York, 1996) Chap. 3.

[76] See, for example, J. E. Annett, Superconductivity, Superfluids and Condensates (Oxford University Press, Oxford, 2004) Chap. 5.

[77] J. M. Blatt, Theory of Superconductivity (Academic Press, New York, 1964) Chap. 3.

[78] L. V. Keldysh and A. N. Kozlov, Sov. Phys. JETP 27, 521 (1968).

[79] As is well known [65, 75], the fluctuation of the particle number for the BCS ground state is proportional to the gap parameter at zero temperature and to the density of states at the Fermi level. Since the density of states is proportional to the volume of the system, the value of the above fluctuation is $O(N)$. Similarly, the fluctuation of the particle number for the coherent state of the boson system is rigorously equal to the number of the constituent boson [76].

[80] As shown in Sec. II, the OPSS is given by the average of $\psi(\mathbf{r} \zeta) \psi\left(\mathbf{r}^{\prime} \zeta^{\prime}\right)$. It is obvious that the particle number conservation is broken when this order parameter has nonzero value.

[81] M. Higuchi and K. Higuchi, Phys. Rev. B 69, 035113 (2004).

[82] K. Higuchi and M. Higuchi, Phys. Rev. B 69, 165118 (2004).

[83] K. Higuchi and M. Higuchi, Phys. Rev. B 71, 035116 (2005).

[84] K. Higuchi and M. Higuchi, Phys. Rev. A. 79, 022113 (2009).

[85] M. Higuchi and K. Higuchi, Phys. Rev. A. 81, 042505 (2010).

[86] N. D. Mermin, Phys. Rev. 137, A1441 (1965). 